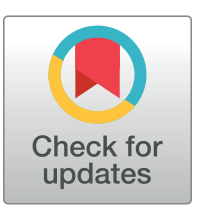

OPEN ACCESS

Citation: Dahal P, Singh-Phulgenda S, Maguire BJ, Harriss E, Ritmeijer K, Alves F, et al. (2021) Visceral Leishmaniasis in pregnancy and vertical transmission: A systematic literature review on the therapeutic orphans. PLoS Negl Trop Dis 15(8): e0009650. https://doi.org/10.1371/journal. pntd.0009650

Editor: Walderez 0. Dutra, Instituto de Ciências Biológicas, Universidade Federal de Minas Gerais, BRAZIL

Received: February 18, 2021

Accepted: July 13, 2021

Published: August 10, 2021

Peer Review History: PLOS recognizes the benefits of transparency in the peer review process; therefore, we enable the publication of all of the content of peer review and author responses alongside final, published articles. The editorial history of this article is available here: https://doi.org/10.1371/journal.pntd.0009650

Copyright: @ 2021 Dahal et al. This is an open access article distributed under the terms of the Creative Commons Attribution License, which permits unrestricted use, distribution, and reproduction in any medium, provided the original author and source are credited.

Data Availability Statement: The database(s) supporting the conclusions of this article are
RESEARCH ARTICLE

\section{Visceral Leishmaniasis in pregnancy and vertical transmission: A systematic literature review on the therapeutic orphans}

\author{
Prabin Dahal $\oplus^{1,2 *}$, Sauman Singh-Phulgenda ${ }^{1,2}$, Brittany J. Maguire $\oplus^{1,2}$, Eli Harriss $\oplus^{3}$, \\ Koert Ritmeijer $\oplus^{4}$, Fabiana Alves $\oplus^{5}$, Philippe J. Guerin $\oplus^{1,2}$, Piero L. Olliaro $\circledast^{2}$ \\ 1 Infectious Diseases Data Observatory (IDDO), Oxford, United Kingdom, 2 Centre for Tropical Medicine \\ and Global Health, Nuffield Department of Medicine, University of Oxford, Oxford, United Kingdom, 3 The \\ Knowledge Centre, Bodleian Health Care Libraries, University of Oxford, Oxford, United Kingdom, \\ 4 Médecins Sans Frontières, Amsterdam, Netherlands, 5 Drugs for Neglected Diseases initiative, Geneva, \\ Switzerland \\ * prabin.dahal@iddo.org
}

\section{Abstract}

\section{Background}

Reports on the occurrence and outcome of Visceral Leishmaniasis $(\mathrm{VL})$ in pregnant women is rare in published literature. The occurrence of $\mathrm{VL}$ in pregnancy is not systematically captured and cases are rarely followed-up to detect consequences of infection and treatment on the pregnant women and foetus.

\section{Methods}

A review of all published literature was undertaken to identify cases of $V L$ infections among pregnant women by searching the following database: Ovid MEDLINE; Ovid Embase; Cochrane Database of Systematic Reviews; Cochrane Central Register of Controlled Trials; World Health Organization Global Index Medicus: LILACS (Americas); IMSEAR (SouthEast Asia); IMEMR (Eastern Mediterranean); WPRIM (Western Pacific); ClinicalTrials.gov; and the WHO International Clinical Trials Registry Platform. Selection criteria included any clinical reports describing the disease in pregnancy or vertical transmission of the disease in humans. Articles meeting pre-specified inclusion criteria and non-primary research articles such as textbook, chapters, letters, retrospective case description, or reports of accidental inclusion in trials were also considered.

\section{Results}

The systematic literature search identified 272 unique articles of which 54 records were included in this review; a further 18 records were identified from additional search of the references of the included studies or from personal communication leading to a total of 72 records (71 case reports/case series; 1 retrospective cohort study; 1926-2020) describing 451 cases of VL in pregnant women. The disease was detected during pregnancy in 398 $(88.2 \%)$, retrospectively confirmed after giving birth in $52(11.5 \%)$, and the time of 
available within the tables and figures presented within the manuscript along with the supplemental files (S1 Data, S2 Data).

Funding: The review was funded by a biomedical resource grant from Wellcome to the Infectious Diseases Data Observatory (Recipient: PJG; ref: 208378/Z/17/Z). The funders had no role in the design and analysis of the research or the decision to publish the work.

Competing interests: The authors have declared that no competing interests exist. identification was not clear in $1(0.2 \%)$. Of the 398 pregnant women whose infection was identified during pregnancy, $346(86.9 \%)$ received a treatment, $3(0.8 \%)$ were untreated, and the treatment status was not clear in the remaining 49 (12.3\%). Of 346 pregnant women, Liposomal amphotericin B (L-AmB) was administered in 202 (58.4\%) and pentavalent antimony (PA) in $93(26.9 \%)$. Outcomes were reported in 176 pregnant women treated with L-AmB with 4 (2.3\%) reports of maternal deaths, 5 (2.8\%) miscarriages, and $2(1.1 \%)$ foetal death/stillbirth. For PA, outcomes were reported in 88 of whom 4 (4.5\%) died, 24 $(27.3 \%)$ had spontaneous abortion, $2(2.3 \%)$ had miscarriages. A total of 26 cases of confirmed, probable or suspected cases of vertical transmission were identified with a median detection time of 6 months (range: $0-18$ months).

\section{Conclusions}

Outcomes of VL treatment during pregnancy is rarely reported and under-researched. The reported articles were mainly case reports and case series and the reported information was often incomplete. From the studies identified, it is difficult to derive a generalisable information on outcomes for pregnant women and babies, although reported data favours the usage of liposomal amphotericin B for the treatment of VL in pregnant women.

\section{Author summary}

Visceral Leishmaniasis (VL) is a neglected tropical disease with an estimated incidence of 50,000 to 90,000 cases in 2019. Women who are susceptible to becoming pregnant or those who are pregnant and lactating are regularly excluded from clinical studies of VL. A specific concern of public health relevance is the little knowledge of the consequences of $\mathrm{VL}$ and its treatment on the mother and the foetus. We did a systematic review of all published literature with an overarching aim of identifying cases of VL in pregnancy and assessing the risk-benefit balance of antileishmanial treatment to the pregnant women and the child. We identified a total of 72 records (1926-2020) describing 451 VL cases in pregnant women. In 398, infection was identified during pregnancy of whom 202 received Liposomal Amphotericin B (L-AmB) and 93 received pentavalent antimony (PA). In studies that reported maternal outcomes, reports of maternal death abortion/spontaneous abortion, and miscarriages were proportionally lower among those who received L-AmB compared to PA (no formal test of significance carried out). A total of 26 cases of confirmed, probable or suspected cases of vertical transmission were identified and the median time to detection was 6 months (range: 0-18 months). Our review brings together scattered observations of VL in pregnant women in the clinical literature and clearly highlights that the disease in pregnancy is under-reported and under-studied. The collated evidence derived mainly from case reports and case series indicate that L-AmB has a favourable safety profile than the antimony regimen and should be the preferred treatment for VL during pregnancy.

\section{Introduction}

Visceral Leishmaniasis (VL) is a neglected tropical disease caused by the parasite Leishmania donovani in Asia and Africa and Leishmania infantum in the Mediterranean Basin, the Middle 
East, Central Asia, South America, and Central America [1]. The disease is transmitted by female sandflies with an estimated 50,000 to 90,000 cases in 2019 [2]. India, Sudan, Brazil, Ethiopia, Kenya, and South Sudan accounted for more than $80 \%$ of the total VL cases reported to the WHO in 2019 [3].

The main clinical features of VL include persistent fever, splenomegaly, weight loss, and anaemia [1]. If untreated, the disease is generally fatal within 2 years [1]. Among those with clinical suspicion of the disease, diagnosis of VL is confirmed through demonstration of the parasites in a tissue aspirate obtained from the spleen, bone marrow, or lymph node. The accuracy of the diagnosis is sample dependent with a sensitivity greater than $90 \%$ for spleen specimen (gold standard approach) and $50-80 \%$ for bone marrow sample, but a splenic aspirate carries a risk of haemorrhage in one per 1,000 procedures [1]. Lipososmal amphotericin $B$ $(\mathrm{L}-\mathrm{AmB})$ is the drug of choice for the treatment of VL in the Indian sub-continent, while a combination of pentavalent antimony and paromomycin is used in Eastern Africa. Blood transfusion may be required before initiation of treatment [4-6] or during the treatment or post-treatment follow-up period $[7,8]$. In the clinical literature, the disease is predominantly described among males $[9,10]$.

A specific concern of public health relevance is the little knowledge of the clinical aspects of $\mathrm{VL}$ and treatment outcomes in pregnant and lactating women [11]. VL in pregnancy presents a number of challenges. During pregnancy, the use of splenic aspirate poses additional risk for the foetus. Pregnant women are also more likely to present with more severe anaemia than non-pregnant women and they are at an increased risk of requiring blood transfusion [11, 12]. During pregnancy, the optimal case management must take into account the consequences of the disease and the therapeutic intervention on the pregnant woman and the foetus [13]. Of note, except amphotericin B, all other available drugs are either contraindicated or subjected to restricted use in pregnant and lactating women and in women of child-bearing age (Table 1) [14-16]. Further complexities arise from potential vertical transmission of the disease either congenitally or through transplacental infection as a result of blood exchange during labour [17-19]. Such vertical transmission can induce in utero death or can be potentially deleterious to the foetus and infant $[16,20,21]$. While vertical transmission of VL is well-studied and established in animal studies, reports in humans are sporadic with observations of clinical manifestation several months post-partum [21-24].

The regulatory restrictions and limited evidence on safety of antileishmanial chemotherapeutics on the mother-foetus pair meant that historically clinicians had to rely on personal experience or limited published case-reports to make a decision regarding treatment. This led to some clinicians delaying the treatment of pregnant women until after delivery, especially when the case was detected closer to the due date $[25,26]$. Others had treated them when the adjudicated risk of VL to the mother outweighed the risk posed by the drug to the mother-foetus pair [27]. Currently liposomal amphotericin B (L-AmB) remains the preferred regimen for the treatment in pregnancy (Table 1). However, pregnant and lactating women are regularly excluded from clinical studies [10] and are considered "therapeutic orphans" [28]. In studies that enrol females of childbearing age, counselling measures are usually set in place to inform the patients regarding the potential teratogenic harms of study drugs and either adoption of suitable contraception methods or observance of abstinence is mandatory [10]. In regular clinical practice and non-clinical trial settings, pregnancy tests and counselling however, might not be done routinely. A study conducted in South Asia found that only one in every six doctors ruled out pregnancy before prescribing miltefosine, a medication which has been contraindicated in pregnancy [29].

Finally, there is a lack of active pregnancy registries for most of the antileishmanial drugs except for miltefosine. In the context of Impavido (Profounda Inc.), the commercial name of 
Table 1. Antileishmanial usage during pregnancy.

\begin{tabular}{|c|c|c|}
\hline Drug & Indication & $\begin{array}{l}\text { FDA category (reviewed in } \\
[33,34])^{\mathbf{a}}\end{array}$ \\
\hline $\begin{array}{l}\text { Pentavalent antimony: Pentostam } \\
\text { (Sodium Stibogluconate) }\end{array}$ & $\begin{array}{l}\text { "Pentavalent antimonials are less safe in pregnancy, as they can result in spontaneous abortion, } \\
\text { preterm deliveries and hepatic encephalopathy in the mother and vertical transmission" } \\
\text {-Source: WHO-2010 [13] } \\
\text { "Do not give during pregnancy unless there is no choice" } \\
\text {-Source: South Sudan country guidelines [35] }\end{array}$ & $\begin{array}{l}\text { C (Risks cannot be ruled } \\
\text { out) }\end{array}$ \\
\hline Amphotericin B deoxycholate & $\begin{array}{l}\text { "Amphotericin B deoxycholate and lipid formulations are the best therapeutic options for } \\
\text { visceral leishmaniasis. No abortions or vertical transmission have been reported in mothers } \\
\text { treated with liposomal amphotericin B" } \\
\text {-Source: WHO-2010 [13] }\end{array}$ & $\begin{array}{l}\text { B (No evidence of risk from } \\
\text { existing studies) }\end{array}$ \\
\hline $\begin{array}{l}\text { Liposomal amphotericin B } \\
\text { (AmBisome) }\end{array}$ & $\begin{array}{l}\text { "Animal studies do not indicate direct or indirect harmful effects with respect to reproductive } \\
\text { toxicity. The safety of AmBisome in pregnant women has not been established. Systemic fungal } \\
\text { infections have been successfully treated in pregnant women with conventional amphotericin B } \\
\text { without obvious effect on the foetus, but the number of cases reported is insufficient to draw any } \\
\text { conclusions on the safety of AmBisome in pregnancy. AmBisome should only be used during } \\
\text { pregnancy if the possible benefits to be derived outweigh the potential risks to the mother and } \\
\text { foetus. It is unknown whether AmBisome is excreted in human breast milk. A decision on } \\
\text { whether to breastfeed while receiving AmBisome should take into account the potential risk to } \\
\text { the child as well as the benefit of breast feeding for the child and the benefit of AmBisome } \\
\text { therapy for the mother" } \\
\text {-Source: The EMC [36] } \\
\text { "Amphotericin B deoxycholate and lipid formulations are the best therapeutic options for } \\
\text { visceral leishmaniasis. No abortions or vertical transmission have been reported in mothers } \\
\text { treated with liposomal amphotericin B" } \\
\text {-Source: The WHO-2010 [13] } \\
\text { This is the first line therapy for treatment against pregnancy in Kenya [37], Ethiopia [38], } \\
\text { Somalia [39], Republic of Sudan [40], South Sudan [35], Uganda [41], and Brazil [42, 43] }\end{array}$ & $\begin{array}{l}\text { B (No evidence of risk from } \\
\text { existing studies) }\end{array}$ \\
\hline Pentamidine & $\begin{array}{l}\text { Contraindicated during the first trimester of pregnancy } \\
\text {-Source: WHO-2010 [13] }\end{array}$ & $\begin{array}{l}\text { C (Risks cannot be ruled } \\
\text { out) }\end{array}$ \\
\hline Miltefosine (IMPAVIDO) & $\begin{array}{l}\text { Contraindicated in pregnancy } \\
\text { "IMPAVIDO may cause fetal harm. Fetal death and teratogenicity, occurred in animals } \\
\text { administered miltefosine at doses lower than the recommended human dose. Do not administer } \\
\text { IMPAVIDO to pregnant women. Obtain a serum or urine pregnancy test in females of } \\
\text { reproductive potential prior to prescribing IMPAVIDO. Advise females of reproductive } \\
\text { potential to use effective contraception during therapy and for } 5 \text { months after therapy" } \\
\text {-Source: Prescribing information for IMPAVIDO [44] } \\
\text { "Miltefosine is potentially embryotoxic and teratogenic and should not be used during } \\
\text { pregnancy. Women of child-bearing age should be tested for pregnancy before treatment and } \\
\text { use effective contraception for } 3 \text { months after treatment" } \\
\text {-Source: WHO-2010 [13] }\end{array}$ & D (Positive evidence of risk) \\
\hline Paromomycin (aminosidine) & $\begin{array}{l}\text { "Ototoxicity in the fetus is the main concern. Insufficient data are available on the use of } \\
\text { paromomycin in pregnant women" } \\
\text {-Source: WHO-2010 [13] } \\
\text { "Paromomycin crosses the placenta and can cause renal and auditory damage in the unborn } \\
\text { child. Paromomycin is excreted in breast milk and adverse effects in the breastfed infant cannot } \\
\text { be excluded." } \\
\text {-Source: National guidelines of Kenya-2017 [37] }\end{array}$ & No category assigned \\
\hline
\end{tabular}

a Silva et al (2013)[33] review is in context of treatment of pregnancy in American tegumentary leishmaniasis. The following definition of FDA category is presented: Category B-remote possibility of foetal harm; animal studies showed no risk to the foetus; there are no studies in humans; Category C- possible harm to the foetus; insufficient controlled studies in humans and animals [33].

https://doi.org/10.1371/journal.pntd.0009650.t001

miltefosine registered to the US medicines regulatory agency (US Food Drug Administration), a pregnancy registry was established to fulfil post marketing requirements [30, 31]. The recruitment of pregnant women as part of the observational study started in 2015 and the study is expected to be completed in 2026, and is estimated to recruit $0-1$ patients per year over the 10 year study period, hence unlikely to generate a large volume of new safety data 
[30]. There are no other active pregnancy registries on exposures to VL treatments from which to derive information on consequences on gestation, mother, foetus, and the newborn. Therefore, to understand the risks and benefits of treatment to the pregnant woman and the child, one must turn to the published literature.

The most comprehensive reviews on VL in pregnant women were conducted in the mid 2000 s [21,32]. We therefore conducted a systematic review of all published literature with an overarching aim of identifying cases of VL in pregnancy. The specific objectives were to assess the risk-benefit balance of antileishmanial therapies to the pregnant woman and the child and to identify the cases of vertical transmission. The review was not limited by language or any interventions.

\section{Material and methods}

\section{Literature search}

A review of all published literature was undertaken on $26^{\text {th }}$ of March 2020 to identify records describing VL in pregnant women or any reports of vertical transmission of the disease in humans by searching the following clinical databases: Ovid MEDLINE; Ovid Embase; Cochrane Database of Systematic Reviews; Cochrane Central Register of Controlled Trials; World Health Organization Global Index Medicus: LILACS (Americas); IMSEAR (South-East Asia); IMEMR (Eastern Mediterranean); WPRIM (Western Pacific); ClinicalTrials.gov; and the WHO International Clinical Trials Registry Platform (ICTRP). The systematic review was conducted in accordance with the Preferred Reporting Items for Systematic-Reviews and Meta-Analyses (PRISMA) guidelines (S1 Text) [45]. In addition, full text screening of the publications indexed in the Infectious Diseases Data Observatory (IDDO) clinical trials library was carried out to identify any description of VL in pregnant women [46]. The references of all included publications were further checked to identify any relevant articles. This review is not registered and the protocol describing the search strategy including search strings, search dates and eligibility criteria for screening is presented in supplemental file (S2 Text).

\section{Study screening}

Study screening was carried out in two stages to identify the studies fulfilling the inclusion and exclusion criteria (S2 Text): title and abstract screening (stage I) and then full-text screening (stage II). As reports on VL in pregnancy are sparse, articles meeting minimal inclusion criteria and non-primary research articles such as opinion pieces, clinical guidelines, textbooks, chapters, correspondences, reports of accidental inclusion in trials, or case reports of unplanned pregnancies during the study follow-up were also considered for comprehensiveness. No restrictions were applied regarding study design, follow-up duration, sample size, region, or the treatment regimen for eligibility of inclusion in this review. Articles that were not in English language (Spanish, Portuguese, Korean, and German) were reviewed using google translation (https://translate.google.co.uk/).

The articles were screened against eligibility criteria by a single reviewer (PD). A second reviewer was consulted (SSP) when the first reviewer could not reliably assess the eligibility. The first reviewer (PD) extracted data from all the eligible records and it was verified by the second reviewer (SSP) (who was not blinded) on all publications included in the review. Any discrepancy in the extracted information was flagged by the second reviewer and the differences were resolved through consensus. Screening and data extraction was carried out on a prospectively designed Excel database (S1 and S2 Data). 


\section{Data extraction}

The following bibliographic information were extracted: study title, name of the first author, year of publication, name of the study site and country. The following maternal and child characteristics were extracted: age of the pregnant woman, period of gestation (or trimester), treatment administered including drug dosage, follow-up duration, the outcome of the treatment for pregnant woman (cured, relapsed, death), and foetal outcomes (abortion, stillbirth, premature birth, healthy born, vertical transmission).

\section{Definitions}

The records were classified as: case report/case series, prospective cohort or retrospective cohort studies. Records describing one or a small group of patients included as a part of prospective (or retrospective) studies in which VL in pregnancy was not of primary focus were considered as case report/case series. Similarly, studies that described a cohort of pregnant women without selection of a non-pregnant comparator group were also considered as case series. Countries were classified into sub-regions according to United Nations designation of geographical regions [47].

\section{Data analysis}

Since the majority of the studies included were either case reports or case series, analysis of data was restricted to presentation of descriptive statistics and meta-analysis was not carried out. Descriptive summaries were presented for the characteristics of the studies included in the review, characteristics of pregnant woman (trimester, gestational age), treatment regimen including dosage and duration, clinical outcomes on the pregnant woman and the child. Graphics were generated using R software [48].

\section{Assessment of risk of bias}

The risk of bias in case report/case series was assessed using a checklist proposed in Murad2018 [49]. The following domains were assessed: patient selection, ascertainment of exposure, follow-up of pregnant women until delivery to record pregnancy related outcomes, and completeness of reporting of treatment and outcome status. Methodology and definitions adopted for assessment of risk of bias is presented in S1 Table. Briefly, bias in ascertainment of exposure was considered high if VL diagnosis was based solely on clinical features. A single case report was considered to be at a high risk of selection bias whereas a series of cases selected based on an audit of complete records over a study period was considered to be at a low risk of selection bias. If a study followed-up the pregnant women until birth and reported outcomes of the treatment for the mother-foetus pair, then follow-up was considered adequate to assess pregnancy-related outcomes. Bias in reporting of results was assessed based on the completeness of reporting on treatment status and clinical outcomes (See S1 Table for further explanations). For cohort studies (prospective or retrospective), risk of bias was assessed using The Newcastle-Ottawa scale. Two authors (PD, SSP) assessed the risk of bias in the studies included.

\section{Results}

We identified 395 records from the literature searches up until $26^{\text {th }}$ of March 2020, of which 272 were unique after removing duplicate entries. Of the 272 unique records, 99 were excluded at title and abstract screening stage leaving 173 records for full-text assessment of which 54 met the eligibility criteria for inclusion in the review (Fig 1). An additional 18 records were identified by searching the references of the eligible records or through personal 


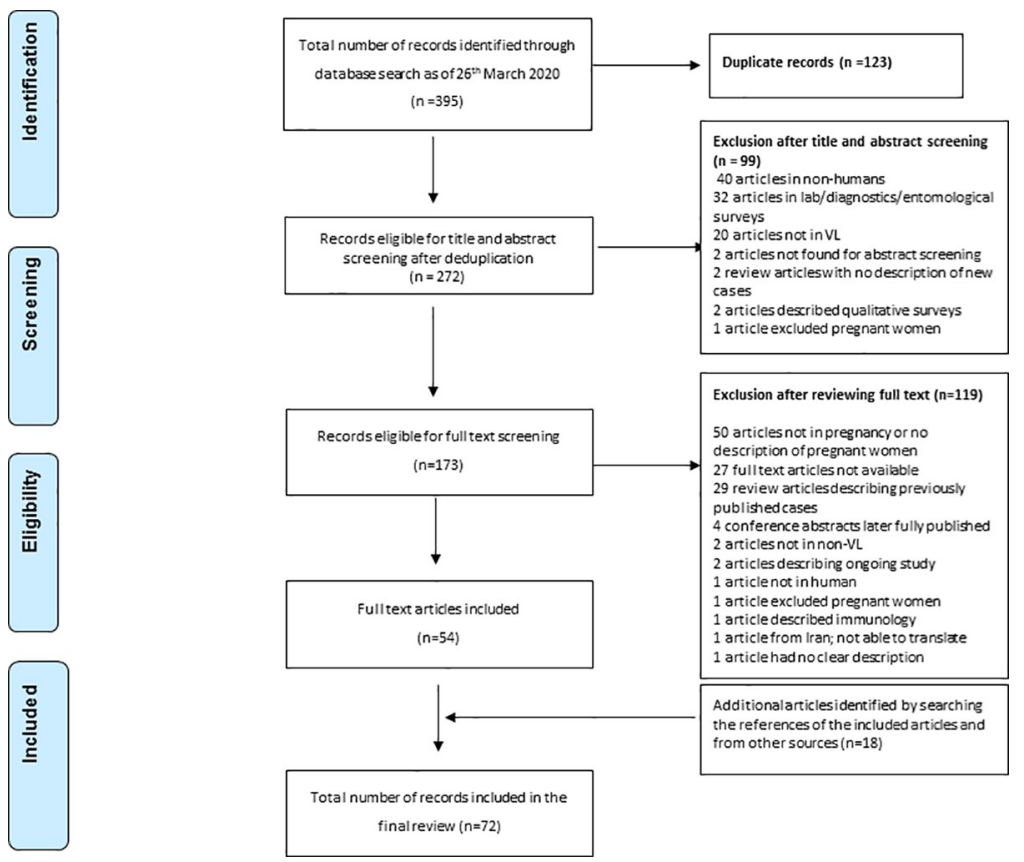

Fig 1. Preferred Reporting Items for Systematic Reviews and Meta-Analyses (PRISMA) flow diagram of publications screened.

https://doi.org/10.1371/journal.pntd.0009650.g001

communication. A total of 72 records published from 1926 through 2020 were included in this review of which 71 were case-reports or case-series and one was a retrospective cohort study with non-pregnant patient as a comparative group (Table 2). Further details on the studies included in this review are presented in supplemental files (S1 and S2 Data).

\section{Spatial and temporal distribution}

A total of 21 (29.2\%) records were from Europe (1926-2019), 23 (31.9\%) from Southern Asia (1955-2018), 13 (18.1\%) from South America (1986-2019), 8 (11.1\%) from Northern Africa (1988-2018), 5 (6.9\%) from Eastern Africa (1984-2020), and 1 (1.4\%) record each were from Eastern Asia (1928) and Western Asia (1979). There were 18 (25.0\%) records from India, 13 (18.1\%) from Brazil, 8 (11.1\%) from Sudan, and further breakdown by country is presented in Fig 2 (left panel). There were 64 (88.9\%) records in English language, 7 (9.7\%) in Portuguese, and $1(1.4 \%)$ in French. The 72 records included in this review described 451 cases of VL in pregnant women, of whom 159 (35.3\%) were from Sudan, 113 (25.1\%) from South Sudan, 84 (18.6\%) from India, 23 (5.1\%) from Bangladesh, 20 (4.4\%) from Brazil, 12 (2.7\%) from Italy, 10 (2.2\%) from Uganda, and the rest of the breakdown is presented in Fig 2 (right panel). Overall, there were $10(13.9 \%)$ records published from 1926 through 1990, 14 (19.4\%) from 1991 through 2000, 22 (30.6\%) from 2001 through 2010, and 26 (36.1\%) from 2011 through 2020 (Table 2).

\section{Treatment regimens}

Of the 451 pregnant women identified, the disease was detected during pregnancy in 398 (88.2\%), retrospectively confirmed after giving birth in 52 (11.5\%), and the time of identification was not clear in one $(0.2 \%)$. Of the 398 pregnant women whose infection was identified during pregnancy, $346(86.9 \%)$ received a treatment, $3(0.8 \%)$ were untreated, and the 
Table 2. Description of reported cases of visceral leishmaniasis in pregnant or lactating women.

\begin{tabular}{|c|c|c|c|c|c|c|}
\hline Author-year & Country & $\begin{array}{l}\text { Time of detection/ } \\
\text { description }\end{array}$ & $\begin{array}{l}\text { Number of } \\
\text { pregnant } \\
\text { woman/ } \\
\text { women }\end{array}$ & Trimester & $\begin{array}{l}\text { Description of treatment in } \\
\text { pregnancy }\end{array}$ & Pregnancy outcome \\
\hline Low-1926 [19] & UK & During pregnancy & 1 & $3^{\text {rd }}$ & Urea stibamine & Normal delivery \\
\hline Hindle-1928 [66] & China & $\begin{array}{l}\text { Retrospectively } \\
\text { suspected }\end{array}$ & 1 & $\begin{array}{l}\text { Not applicable } \\
\text { (retrospective) }\end{array}$ & No information & No description \\
\hline Hindle-1928 [66] & China & $\begin{array}{l}\text { Retrospectively } \\
\text { suspected }\end{array}$ & 1 & $\begin{array}{l}\text { Not applicable } \\
\text { (retrospective) }\end{array}$ & No information & No description \\
\hline Banerji-1955 [56] & India & During pregnancy & 1 & $2^{\text {nd }}$ & $\begin{array}{l}\text { Treated with } 10 \text { intravenous } \\
\text { Urea stibamine }\end{array}$ & Remission of fever \\
\hline El-Saaran-1979 [54] & UAE & During pregnancy & 1 & $2^{\text {nd }}$ & $\begin{array}{l}\text { Pentostam: } 6 \mathrm{ml} \text { intravenous } \\
\text { dose daily for ten days, at } \\
\text { intervals of ten days to a total of } \\
180 \mathrm{ml}\end{array}$ & $\begin{array}{l}\text { Did not recover; splenectomy } \\
\text { performed after birth }\end{array}$ \\
\hline Rees-1984 [67] & Kenya & During pregnancy & 1 & No information & Pentostam & No information \\
\hline Blanc-1984 [68] & France & $\begin{array}{l}\text { Retrospectively } \\
\text { identified }\end{array}$ & 1 & $\begin{array}{l}\text { Not applicable } \\
\text { (retrospective) }\end{array}$ & $\begin{array}{l}\text { Treatment with N- } \\
\text { methylglucamine (antimony) for } \\
10 \text { days }\end{array}$ & Normal delivery \\
\hline Badaro-1986 [69] & Brazil & During pregnancy & 1 & $3^{\text {rd }}$ & $\begin{array}{l}\text { Untreated (posthumous } \\
\text { diagnosis) }\end{array}$ & Mother died 5 weeks later \\
\hline Mittal-1987 [62] & Indian & During pregnancy & 1 & $3^{\text {rd }}$ & Drug name not stated & Normal term delivery \\
\hline Nyakundi-1988 [70] & Sudan & During pregnancy & 1 & Not clear & Unclear & $\begin{array}{l}\text { Premature birth at } 6 \text { months of } \\
\text { gestation }\end{array}$ \\
\hline Yadav-1989 [71] & India & $\begin{array}{l}\text { Retrospectively } \\
\text { identified (symptoms } \\
\text { shown during } \\
\text { pregnancy) }\end{array}$ & 1 & $2^{\text {nd }}$ & $\begin{array}{l}\text { Not treated (herbal medicine was } \\
\text { given) }\end{array}$ & Normal and uneventful delivery \\
\hline Aggarwal-1991 [72] & India & During pregnancy & 1 & $2^{\text {nd }}$ (6 months) & Sodium antimony gluconate & $\begin{array}{l}\text { Healthy baby delivered at full } \\
\text { term }\end{array}$ \\
\hline Aggarwal -1991 [72] & India & During pregnancy & 2 & No information & No information & No information \\
\hline Elamin1992 [17] & Sudan & During pregnancy & 1 & $3^{\text {rd }}$ & Drug name not stated & Normal delivery \\
\hline Eltoum-1992 [18] & Sudan & During pregnancy & 1 & $2^{\text {nd }}$ & SSG: $10 \mathrm{mg} / \mathrm{kg} /$ daily for 30 days & Normal delivery \\
\hline Eltoum-1992 [18] & Sudan & During pregnancy & 1 & $2^{\text {nd }}$ & Not clear & $\begin{array}{l}\text { Abortion of a female foetus ( } 5 \\
\text { months old) }\end{array}$ \\
\hline Seaman-1993 [27] & Sudan & During pregnancy & 3 & Not clear & SSG $20 \mathrm{mg} / \mathrm{kg} /$ day for 30 days & No information \\
\hline Seaman-1993 [27] & Sudan & During pregnancy & 3 & Not clear & $\begin{array}{l}\text { SSG + aminosidine }(20 \mathrm{mg} / \mathrm{kg} / \\
\text { day SSG for } 17 \text { days }+15 \mathrm{mg} / \mathrm{kg} \\
\text { of aminosidine for } 17 \text { days })\end{array}$ & No information \\
\hline Thakur-1993 [73] & India & During pregnancy & 1 & $2^{\text {nd }}$ & $\begin{array}{l}\text { Amphotericin B }(1 \mathrm{mg} / \mathrm{kg} \text { body } \\
\text { weight daily starting with } 0.5 \mathrm{mg} / \\
\mathrm{kg} \text { body weight till a total dose of } \\
20 \mathrm{mg} / \mathrm{kg})\end{array}$ & Normal delivery \\
\hline Thakur-1993 [73] & India & During pregnancy & 1 & $2^{\text {nd }}$ & $\begin{array}{l}\text { Amphotericin B }(1 \mathrm{mg} / \mathrm{kg} \text { body } \\
\text { weight daily starting with } 0.5 \mathrm{mg} / \\
\mathrm{kg} \text { body weight till a total dose of } \\
20 \mathrm{mg} / \mathrm{kg})\end{array}$ & Normal delivery \\
\hline Thakur-1993 [73] & India & During pregnancy & 1 & $1^{\text {st }}$ & $\begin{array}{l}\text { Amphotericin B }(1 \mathrm{mg} / \mathrm{kg} \text { body } \\
\text { weight daily starting with } 0.5 \mathrm{mg} / \\
\mathrm{kg} \text { body weight till a total dose of } \\
20 \mathrm{mg} / \mathrm{kg})\end{array}$ & Normal delivery \\
\hline Thakur-1993 [73] & India & During pregnancy & 1 & $\begin{array}{l}1^{\text {st }} \text { (febrile } \\
\text { episode } 40 \text { days } \\
\text { after conception) }\end{array}$ & $\begin{array}{l}\text { Amphotericin B }(1 \mathrm{mg} / \mathrm{kg} \text { body } \\
\text { weight daily starting with } 0.5 \mathrm{mg} / \\
\mathrm{kg} \text { body weight till a total dose of } \\
20 \mathrm{mg} / \mathrm{kg})\end{array}$ & Normal delivery \\
\hline Thakur-1993 [73] & India & During pregnancy & 1 & $2^{\text {nd }}$ & $\begin{array}{l}\text { Amphotericin B }(1 \mathrm{mg} / \mathrm{kg} \text { body } \\
\text { weight daily starting with } 0.5 \mathrm{mg} / \\
\mathrm{kg} \text { body weight till a total dose of } \\
20 \mathrm{mg} / \mathrm{kg})\end{array}$ & Normal delivery \\
\hline
\end{tabular}


Table 2. (Continued)

\begin{tabular}{|c|c|c|c|c|c|c|}
\hline Author-year & Country & $\begin{array}{l}\text { Time of detection/ } \\
\text { description }\end{array}$ & $\begin{array}{l}\text { Number of } \\
\text { pregnant } \\
\text { woman/ } \\
\text { women }\end{array}$ & Trimester & $\begin{array}{l}\text { Description of treatment in } \\
\text { pregnancy }\end{array}$ & Pregnancy outcome \\
\hline Giri-1993 [74] & India & During pregnancy & 1 & $3^{\text {rd }}$ & Amphotericin B & Normal term delivery \\
\hline Gradoni-1994 [75] & Italy & During pregnancy & 1 & $2^{\text {nd }}$ & $\mathrm{L}-\mathrm{AmB}$ total dose of $18 \mathrm{mg} / \mathrm{kg}$ & Normal delivery \\
\hline Gradoni-1994 [75] & Italy & $\begin{array}{l}\text { Retrospectively } \\
\text { identified (treated after } \\
\text { delivery) }\end{array}$ & 1 & $\begin{array}{l}\text { Not applicable } \\
\text { (retrospective) }\end{array}$ & $\begin{array}{l}\text { Untreated (diagnosed after } \\
\text { birth); treated with } 18 \mathrm{mg} / \mathrm{kg} / \text { day } \\
\text { PA after birth }\end{array}$ & Normal delivery \\
\hline Jeronimo-1994 [76] & Brazil & During pregnancy & 1 & No information & $\begin{array}{l}\text { Meglumine antimoniate }(20 \mathrm{mg} / \\
\mathrm{kg} / \text { day for } 20 \text { days })\end{array}$ & No information \\
\hline Bano -1994 [61] & India & During pregnancy & 1 & $\begin{array}{l}1^{\text {st }}(12 \text { weeks } \\
\text { pregnant })\end{array}$ & $\begin{array}{l}\text { Trimothoprim, sulphadiazine } \\
\text { and tinidazole }\end{array}$ & $\begin{array}{l}\text { Normal full term delivery of a } \\
\text { healthy baby }\end{array}$ \\
\hline Utili-1995 [23] & Italy & During pregnancy & 1 & $2^{\text {nd }}$ & $\begin{array}{l}\text { Meglumine antimoniate ( } 12 \mathrm{mg} / \\
\mathrm{kg} \text { for } 20 \text { days) }\end{array}$ & $\begin{array}{l}\text { Normal term birth (patient } \\
\text { delivered a baby weighing } 4.2 \mathrm{~kg} \\
\text { at } 41 \text { weeks of pregnancy) }\end{array}$ \\
\hline Sharma-1996 [77] & India & $\begin{array}{l}\text { Retrospectively } \\
\text { identified }\end{array}$ & 1 & $\begin{array}{l}\text { Not applicable } \\
\text { (retrospective) }\end{array}$ & Untreated & Normal delivery \\
\hline Thakur-1998 [78] & India & During pregnancy & 1 & No information & $\begin{array}{l}\text { Amphotericin B deoxycholate } \\
\text { (total dose } 20 \mathrm{mg} / \mathrm{kg} \text { ) }\end{array}$ & Normal delivery \\
\hline Thakur-1998 [78] & India & During pregnancy & 1 & No information & $\begin{array}{l}\text { Amphotericin B deoxycholate } \\
\text { (total dose } 20 \mathrm{mg} / \mathrm{kg} \text { ) }\end{array}$ & Normal delivery \\
\hline Thakur-1999 [5] & India & During pregnancy & 1 & No information & $\begin{array}{l}\text { Amphotericin B (total dose } 20 \\
\mathrm{mg} / \mathrm{kg} \text { ) }\end{array}$ & Normal delivery \\
\hline Thakur-1999 [5] & India & During pregnancy & 1 & No information & $\begin{array}{l}\text { Amphotericin B (total dose } 20 \\
\mathrm{mg} / \mathrm{kg} \text { ) }\end{array}$ & Normal delivery \\
\hline Thakur-1999 [5] & India & During pregnancy & 1 & No information & $\begin{array}{l}\text { Amphotericin B (total dose } 20 \\
\mathrm{mg} / \mathrm{kg} \text { ) }\end{array}$ & Normal delivery \\
\hline Thakur-1999 [5] & India & During pregnancy & 1 & No information & $\begin{array}{l}\text { Amphotericin B (total dose } 20 \\
\mathrm{mg} / \mathrm{kg} \text { ) }\end{array}$ & Normal delivery \\
\hline Meinecke-1999 [79] & Germany & $\begin{array}{l}\text { Retrospectively } \\
\text { identified }\end{array}$ & 1 & $\begin{array}{l}\text { Not applicable } \\
\text { (retrospective) }\end{array}$ & $\begin{array}{l}\text { Untreated (retrospective } \\
\text { identification) }\end{array}$ & $\begin{array}{l}\text { Complicated pregnancy with } \\
\text { febrile gastroenteritis; birth } \\
\text { weight was } 3,720 \mathrm{~g}\end{array}$ \\
\hline Vianna-2001 [80] & Brazil & $\begin{array}{l}\text { Retrospectively } \\
\text { identified }\end{array}$ & 2 & $\begin{array}{l}\text { Not applicable } \\
\text { (retrospective) }\end{array}$ & $\begin{array}{l}\text { Untreated (retrospective } \\
\text { identification) }\end{array}$ & One preterm birth \\
\hline Kumar-2001 [25] & India & During pregnancy & 1 & $3^{\text {rd }}$ & $\begin{array}{l}\text { Untreated (treatment deferred } \\
\text { until birth) }\end{array}$ & $\begin{array}{l}\text { Intrauterine growth retardation; } \\
\text { small for age baby; Emergency } \\
\text { C-section required }\end{array}$ \\
\hline Dereure-2003 [81] & France & $\begin{array}{l}\text { During pregnancy } \\
\text { (routine check-up) }\end{array}$ & 1 & $2^{\text {nd }}$ & $\begin{array}{l}\mathrm{L}-\mathrm{AmB}(3 \mathrm{mg} / \mathrm{kg} \text { daily for five } \\
\text { days; a } 6 \text { th injection } 10 \text { days } \\
\text { later) }\end{array}$ & Normal term birth \\
\hline Caldas-2003 [82] & Brazil & During pregnancy & 1 & $1^{\text {st }}$ & $\begin{array}{l}\text { Amphotericin B (1mg/kg for } 14 \\
\text { days) }\end{array}$ & Normal term birth \\
\hline Silveira-2003 [55] & Brazil & During pregnancy & 1 & $2^{\text {nd }}$ & $\begin{array}{l}\text { Meglumine antimoniate }(850 \mathrm{mg} / \\
\text { day for } 20 \text { days) }\end{array}$ & Premature birth \\
\hline Pagliano-2003 [83] & Italy & During pregnancy & 2 & No information & $\mathrm{L}-\mathrm{AmB}$ & Normal delivery \\
\hline Kumar-2004 [84] & Iran & $\begin{array}{l}\text { Retrospectively } \\
\text { identified (after death } \\
\text { of mother-child) }\end{array}$ & 1 & $3^{\text {rd }}$ & $\begin{array}{l}\text { Untreated (posthumous } \\
\text { diagnosis) }\end{array}$ & Death \\
\hline Pagliano-2005 [21] & Italy & During pregnancy & 1 & Not clear & $\begin{array}{l}\mathrm{L}-\mathrm{AmB}(3 \mathrm{mg} / \mathrm{kg} \text { at days } 1-5 \& 3 \\
\mathrm{mg} / \mathrm{kg} \text { at day } 10)\end{array}$ & Healthy term birth \\
\hline Pagliano-2005 [21] & Italy & During pregnancy & 1 & Not clear & $\begin{array}{l}\mathrm{L}-\mathrm{AmB}(3 \mathrm{mg} / \mathrm{kg} \text { at days } 1-5 \& 3 \\
\mathrm{mg} / \mathrm{kg} \text { at day } 10)\end{array}$ & Healthy term birth \\
\hline Pagliano-2005 [21] & Italy & During pregnancy & 1 & Not clear & $\begin{array}{l}\mathrm{L}-\mathrm{AmB}(3 \mathrm{mg} / \mathrm{kg} \text { at days } 1-5 \& 3 \\
\mathrm{mg} / \mathrm{kg} \text { at day } 10)\end{array}$ & Healthy term birth \\
\hline Pagliano-2005 [21] & Italy & During pregnancy & 1 & Not clear & $\begin{array}{l}\mathrm{L}-\mathrm{AmB}(3 \mathrm{mg} / \mathrm{kg} \text { at days } 1-5 \& 3 \\
\mathrm{mg} / \mathrm{kg} \text { at day } 10)\end{array}$ & Healthy term birth \\
\hline
\end{tabular}


Table 2. (Continued)

\begin{tabular}{|c|c|c|c|c|c|c|}
\hline Author-year & Country & $\begin{array}{l}\text { Time of detection/ } \\
\text { description }\end{array}$ & $\begin{array}{l}\text { Number of } \\
\text { pregnant } \\
\text { woman/ } \\
\text { women }\end{array}$ & Trimester & $\begin{array}{l}\text { Description of treatment in } \\
\text { pregnancy }\end{array}$ & Pregnancy outcome \\
\hline Pagliano-2005 [21] & Italy & During pregnancy & 1 & Not clear & $\begin{array}{l}\mathrm{L}-\mathrm{AmB}(3 \mathrm{mg} / \mathrm{kg} \text { at days } 1-5 \& 3 \\
\mathrm{mg} / \mathrm{kg} \text { at day } 10)\end{array}$ & Healthy term birth \\
\hline $\begin{array}{l}\text { Figueiró Filho-2005 } \\
\text { [85] }\end{array}$ & Brazil & During pregnancy & 1 & $3^{\text {rd }}$ & $\begin{array}{l}\mathrm{L}-\mathrm{AmB}(1 \mathrm{mg} / \mathrm{kg} / \text { day for } 21 \\
\text { days })\end{array}$ & $\begin{array}{l}\text { Normal birth at } 38 \text { weeks with } \\
\text { baby weighing } 2,995 \mathrm{~g}\end{array}$ \\
\hline Mueller-2006 [59] & Sudan & During pregnancy & 23 & $\begin{array}{l}11 \text { in } 1^{\text {st }} \\
8 \text { in } 2^{\text {nd }} \\
4 \text { in } 3^{\text {rd }}\end{array}$ & SSG: $20 \mathrm{mg} / \mathrm{kg}$ for 30 days & $\begin{array}{l}13 \text { spontaneous abortion during } \\
\text { days } 13 \text { to } 30 \text { of SSG; } 1 \\
\text { spontaneous abortion prior to } \\
\text { treatment; } 1 \text { healthy baby born; } \\
\text { remaining } 8 \text { still pregnant at } \\
\text { discharge }\end{array}$ \\
\hline Mueller-2006 [59] & Sudan & During pregnancy & 4 & $\begin{array}{l}2 \text { in } 2^{\text {nd }} \\
\text { and } 2 \text { in } 3^{\text {rd }}\end{array}$ & $\begin{array}{l}\mathrm{L}-\mathrm{AmB}+\text { SSG (AmBisome } 3-7 \\
\mathrm{mg} / \mathrm{kg} \text { daily on days } 1,6,11 \text { and } \\
16 \text { (or on days } 1,2,3,4,10 \text {, and } 15 \text { ), } \\
\text { followed by } 20 \mathrm{mg} / \mathrm{kg} \mathrm{SSG} \mathrm{IM} \\
\text { once daily for } 30 \mathrm{~d} \text { ) }\end{array}$ & $\begin{array}{l}1 \text { healthy baby born; remaining } 3 \\
\text { were still pregnant at discharge }\end{array}$ \\
\hline Mueller-2006 [59] & Sudan & During pregnancy & 12 & $\begin{array}{l}2 \text { in } 1^{\text {st }} \\
6 \text { in } 2^{\text {nd }} \\
4 \text { in } 3^{\text {rd }}\end{array}$ & $\begin{array}{l}\mathrm{L}-\mathrm{AmB} \text { (AmBisome } 3-7 \mathrm{mg} / \mathrm{kg} \\
\text { daily on days } 1,6,11 \text { and } 16 \text { (or } \\
\text { on days } 1,2,3,4,10 \text {, and } 15 \text { ) }\end{array}$ & $\begin{array}{l}\text { Premature birth }(\mathrm{n}=1) \text {; two } \\
\text { healthy babies; remaining } 9 \text { still } \\
\text { pregnant at discharge }\end{array}$ \\
\hline Boehme-2006 [86] & Germany & $\begin{array}{l}\text { Possibly before } \\
\text { pregnancy }\end{array}$ & 1 & $\begin{array}{l}\text { Not applicable } \\
\text { (retrospective) }\end{array}$ & $\begin{array}{l}\text { No treatment given } \\
\text { (retrospective speculation) }\end{array}$ & $\begin{array}{l}\text { Spontaneous birth at } 39 \text { weeks of } \\
\text { gestation and healthy baby } \\
\text { delivered }\end{array}$ \\
\hline Vieira-2007 [64] & Brazil & During pregnancy & 1 & $3^{\text {rd }}$ & Untreated & Baby died 2 months after birth \\
\hline Topno-2008 [88] & India & During pregnancy & 1 & $2^{\text {nd }}$ & $\begin{array}{l}\text { Amphotericin B (15 infusions of } \\
1 \mathrm{mg} / \mathrm{kg})\end{array}$ & Normal term birth \\
\hline Topno-2008 [88] & India & During pregnancy & 1 & $2^{\text {nd }}$ & $\begin{array}{l}\text { Amphotericin B (15 infusions of } \\
1 \mathrm{mg} / \mathrm{kg} \text { ) }\end{array}$ & Normal term birth \\
\hline Topno-2008 [88] & India & During pregnancy & 1 & $3^{\text {rd }}$ & $\begin{array}{l}\text { Amphotericin B (15 infusions of } \\
1 \mathrm{mg} / \mathrm{kg})\end{array}$ & Normal term birth \\
\hline Topno-2008 [88] & India & During pregnancy & 1 & $3^{\text {rd }}$ & $\begin{array}{l}\text { Amphotericin B (15 infusions of } \\
1 \mathrm{mg} / \mathrm{kg})\end{array}$ & Normal term birth \\
\hline $\begin{array}{l}\text { Figueiró-Filho-2008 } \\
\text { [57] }\end{array}$ & Brazil & During pregnancy & 1 & $\begin{array}{l}\text { Not clear } \\
\text { (between } 28 \pm 7.8 \\
\text { weeks) }\end{array}$ & $\begin{array}{l}\text { Amphotericin B deoxycholate (1 } \\
\mathrm{mg} / \mathrm{kg} / \text { day for } 20 \text { days) }\end{array}$ & $\begin{array}{l}\text { One maternal death after } 7 \text { days } \\
\text { of treatment due to } \\
\text { haemorrhagic complications } \\
\text { occurring after delivery }\end{array}$ \\
\hline $\begin{array}{l}\text { Figueiró-Filho-2008 } \\
\text { [57] }\end{array}$ & Brazil & During pregnancy & 1 & $\begin{array}{l}\text { Not clear } \\
\text { (between } 28 \pm 7.8 \\
\text { weeks) }\end{array}$ & $\begin{array}{l}\text { Amphotericin B deoxycholate (1 } \\
\mathrm{mg} / \mathrm{kg} / \text { day for } 20 \text { days) }\end{array}$ & No information \\
\hline $\begin{array}{l}\text { Figueiró-Filho-2008 } \\
\text { [57] }\end{array}$ & Brazil & During pregnancy & 1 & $\begin{array}{l}\text { Not clear } \\
\text { (between } 28 \pm 7.8 \\
\text { weeks) }\end{array}$ & $\begin{array}{l}\mathrm{L}-\mathrm{AmB}(3 \mathrm{mg} / \mathrm{kg} / \text { day for } 20 \\
\text { days) }\end{array}$ & No information \\
\hline $\begin{array}{l}\text { Figueiró-Filho-2008 } \\
\text { [57] }\end{array}$ & Brazil & During pregnancy & 1 & $\begin{array}{l}\text { Not clear } \\
\text { (between } 28 \pm 7.8 \\
\text { weeks) }\end{array}$ & $\begin{array}{l}\mathrm{L}-\mathrm{AmB}(3 \mathrm{mg} / \mathrm{kg} / \text { day for } 20 \\
\text { days) }\end{array}$ & No information \\
\hline $\begin{array}{l}\text { Figueiró-Filho-2008 } \\
\text { [57] }\end{array}$ & Brazil & $\begin{array}{l}\text { Retrospectively } \\
\text { confirmed (After } \\
\text { giving birth) }\end{array}$ & 1 & $\begin{array}{l}\text { Not clear } \\
\text { (between } 28 \pm 7.8 \\
\text { weeks) }\end{array}$ & $\begin{array}{l}\text { Untreated during pregnancy } \\
\text { (Diagnosed after birth and given } \\
\text { SSG: } 20 \mathrm{mg} / \mathrm{kg} / \text { day for } 20 \text { days) }\end{array}$ & No information \\
\hline Lorenzi-2008 [89] & UK & $\begin{array}{l}\text { Retrospectively } \\
\text { identified }\end{array}$ & 1 & $\begin{array}{l}\text { Not applicable } \\
\text { (retrospective) }\end{array}$ & $\begin{array}{l}\text { Untreated during pregnancy } \\
\text { (Diagnosed after } 3 \text { years and } \\
\text { treated with amphoteriicn B) }\end{array}$ & Miscarriage \\
\hline Adam-2009 [12] & Sudan & During pregnancy & 42 & $\begin{array}{l}9 \text { in } 1^{\text {st }} \\
21 \text { in } 2^{\text {nd }} \\
12 \text { in } 3^{\text {rd }}\end{array}$ & $\begin{array}{l}\text { SSG: } 20 \mathrm{mg} / \mathrm{kg} \text { SSG once daily } \\
\text { IM for } 30 \text { days }\end{array}$ & $\begin{array}{l}\text { Miscarriage in first trimester } \\
(\mathrm{n}=2) \text {; death due to hepatic } \\
\text { encephalopathy }(\mathrm{n}=4) \text {; preterm } \\
\text { birth }(\mathrm{n}=2)\end{array}$ \\
\hline Mueller-2009 [63] & Uganda & During pregnancy & 10 & No information & $\begin{array}{l}\text { PA or amphotericin b } \\
\text { deoxycholate }\end{array}$ & 2 maternal deaths \\
\hline
\end{tabular}


Table 2. (Continued)

\begin{tabular}{|c|c|c|c|c|c|c|}
\hline Author-year & Country & $\begin{array}{l}\text { Time of detection/ } \\
\text { description }\end{array}$ & $\begin{array}{l}\text { Number of } \\
\text { pregnant } \\
\text { woman/ } \\
\text { women }\end{array}$ & Trimester & $\begin{array}{l}\text { Description of treatment in } \\
\text { pregnancy }\end{array}$ & Pregnancy outcome \\
\hline $\begin{array}{l}\text { Papageorgiou-2010 } \\
\text { [51] }\end{array}$ & Greece & $\begin{array}{l}\text { Confirmed few days } \\
\text { before labour }\end{array}$ & 1 & $3^{\text {rd }}$ & $\begin{array}{l}\mathrm{L}-\mathrm{AmB}(4 \mathrm{mg} / \mathrm{kg} \text { on } 6 \\
\text { consecutive days and repeated } \\
\text { doses at days } 14 \text { and } 21)\end{array}$ & No information \\
\hline Miah-2010 [26] & Bangladesh & During pregnancy & 11 & $2^{\text {nd }}$ & SAG (20 mg/kg for 30 days) & Abortion $(\mathrm{n}=11)$ \\
\hline Miah-2010 [26] & Bangladesh & During pregnancy & 5 & $3^{\text {rd }}$ & SAG (20 mg/kg for 30 days) & Good outcome $(n=5)$ \\
\hline Zinchuk -2010 [52] & Ukraine & During pregnancy & 1 & $3^{\text {rd }}$ & $\begin{array}{l}\mathrm{L}-\mathrm{AmB}(3 \mathrm{mg} / \mathrm{kg} \text { days } 1-5 \\
\text { followed by a single dose } 3 \mathrm{mg} / \mathrm{kg} \\
\text { on day } 10)\end{array}$ & $\begin{array}{l}\text { Delivery by elective C-section at } \\
38 \text { weeks of gestation; baby birth } \\
\text { weight of } 2,800 \mathrm{~g}\end{array}$ \\
\hline Sinha-2010 [90] & India & During pregnancy & 3 & No information & $\begin{array}{l}\mathrm{L}-\mathrm{AmB}(5 \mathrm{mg} / \mathrm{kg} \text { on days } 0,1,4 \text {, } \\
\text { and } 9)\end{array}$ & $\begin{array}{l}\text { Not described (successful } \\
\text { treatment) }\end{array}$ \\
\hline Haque-2010 [91] & Bangladesh & $\begin{array}{l}\text { Retrospectively } \\
\text { identified }\end{array}$ & 1 & $\begin{array}{l}\text { Not applicable } \\
\text { (retrospective) }\end{array}$ & Untreated during pregnancy & $\begin{array}{l}\text { Vertical transmission identified } \\
\text { at } 15 \text { days of birth }\end{array}$ \\
\hline Ritmeijer-2011 [92] & Ethiopia & During pregnancy & 1 & No information & L-AmB (6 infusions of $5 \mathrm{mg} / \mathrm{kg}$ ) & Good response to treatment \\
\hline Ritmeijer-2011 [92] & Ethiopia & During pregnancy & 1 & No information & $\mathrm{L}-\mathrm{AmB}$ (6 infusions of $5 \mathrm{mg} / \mathrm{kg}$ ) & Good response to treatment \\
\hline Sinha-2011 [60] & India & During pregnancy & 3 & No information & $\begin{array}{l}\text { Paromomycin }(11 \mathrm{mg} / \mathrm{kg} / \text { day for } \\
21 \text { days })\end{array}$ & Normal delivery \\
\hline Pilaca-2011 [93] & Albania & $\begin{array}{l}\text { Retrospectively } \\
\text { identified (diagnosis } \\
\text { was made on the day } \\
\text { the baby was born) }\end{array}$ & 1 & $3^{\text {rd }}$ & $\begin{array}{l}\text { Untreated [After giving birth: } \\
\text { Glucantime for } 28 \text { days. The } \\
\text { baby was not fed by his mother's } \\
\text { breast. PA given as L-AmB was } \\
\text { not available] }\end{array}$ & Preterm birth \\
\hline $\begin{array}{l}\text { Damodaran-2012 } \\
\text { [94] }\end{array}$ & UK & $\begin{array}{l}\text { Retrospectively } \\
\text { identified }\end{array}$ & 1 & $\begin{array}{l}\text { Not applicable } \\
\text { (retrospective) }\end{array}$ & No information & $\begin{array}{l}\text { Vertical transmission } \\
\text { (suspected) at } 15 \text { months }\end{array}$ \\
\hline Lima-2013 [95] & Brazil & $\begin{array}{l}\text { Retrospectively } \\
\text { identified (After } 5 \text { days } \\
\text { of giving birth) }\end{array}$ & 1 & $\begin{array}{l}\text { Not applicable } \\
\text { (retrospective) }\end{array}$ & $\begin{array}{l}\text { Untreated during pregnancy } \\
\text { (diagnosed after birth); After } \\
\text { diagnosis L-AmB (total dose of } \\
20 \mathrm{mg} / \mathrm{kg} \text { over } 5 \text { days) }\end{array}$ & $\begin{array}{l}\text { Preterm vaginal birth; mother } \\
\text { died on the } 32^{\text {nd }} \text { day after birth }\end{array}$ \\
\hline Lima-2013 [95] & Brazil & During pregnancy & 1 & $1^{\text {st }}$ & $\mathrm{L}-\mathrm{AmB}$ & No information \\
\hline $\begin{array}{l}\text { Mescouto-Borges- } \\
2013 \text { [96] }\end{array}$ & Brazil & $\begin{array}{l}\text { Retrospectively } \\
\text { identified (After giving } \\
\text { birth) }\end{array}$ & 1 & $2^{\text {nd }}$ & $\begin{array}{l}\text { Untreated (diagnosed after } \\
\text { birth); Amphotericin b } \\
\text { deoxycholate } 1 \mathrm{mg} / \mathrm{kg} \text { followed } \\
\text { by IV L-AmB } 3 \mathrm{mg} / \mathrm{kg} / \text { day }\end{array}$ & $\begin{array}{l}\text { Acute foetal distress requiring C- } \\
\text { section delivery; extremely } \\
\text { premature birth }(1,170 \mathrm{~g})\end{array}$ \\
\hline $\begin{array}{l}\text { Mescouto-Borges- } \\
2013 \text { [96] }\end{array}$ & Brazil & During pregnancy & 1 & $2^{\text {nd }}$ & $\begin{array}{l}\text { Untreated (diagnosed after } \\
\text { birth); IV L-AmB given at } 3 \mathrm{mg} / \\
\mathrm{kg} / \text { day for } 7 \mathrm{~d}\end{array}$ & $\begin{array}{l}\text { Acute foetal distress requiring C- } \\
\text { section delivery; premature birth }\end{array}$ \\
\hline Milosevic-2013 [97] & Serbia & $\begin{array}{l}\text { Retrospectively } \\
\text { identified (After } 31 \\
\text { days of giving birth) }\end{array}$ & 1 & $\begin{array}{l}\text { Not applicable } \\
\text { (retrospective) }\end{array}$ & Untreated (diagnosed after birth) & Normal vaginal delivery \\
\hline Salih-2014 [98] & Sudan & During pregnancy & 23 & No information & $\begin{array}{l}\mathrm{L}-\mathrm{AmB}(30 \mathrm{mg} / \mathrm{kg} \text { divided into } \\
10 \mathrm{IV} \text { infusions of } 3 \mathrm{mg} / \mathrm{kg})\end{array}$ & No information \\
\hline Burza-2014 [99] & India & During pregnancy & 49 & No information & AmBisome & No information \\
\hline Bode-2014 [100] & Germany & Not clear & 1 & No information & No information & $\begin{array}{l}\text { Vertical transmission at } 8 \\
\text { months }\end{array}$ \\
\hline $\begin{array}{l}\text { Llamazares-2014 } \\
{[101]}\end{array}$ & Spain & $\begin{array}{l}\text { Retrospectively } \\
\text { identified }\end{array}$ & 1 & $\begin{array}{l}\text { Not applicable } \\
\text { (retrospective) }\end{array}$ & Untreated during pregnancy & Normal delivery \\
\hline Rahman-2014 [102] & Bangladesh & During pregnancy & 1 & No information & L-AmB & Stillbirth baby \\
\hline Colomba-2015 [103] & Italy & $\begin{array}{l}\text { Retrospectively } \\
\text { identified (After } 4 \text { days } \\
\text { of giving birth) }\end{array}$ & 1 & After delivery & $\begin{array}{l}\text { Untreated: (treated with } \mathrm{L}-\mathrm{AmB} \\
3 \mathrm{mg} / \mathrm{kg} / \text { day on days } 1-5 \text { and on } \\
\text { day } 10 \text { ) }\end{array}$ & No information \\
\hline Pawar-2015 [104] & India & During pregnancy & 1 & $2^{\text {nd }}$ & $\begin{array}{l}\text { Amphotericin B deoxycholate } \\
\text { (later switched to liposomal } \\
\text { preparation to minimise } \\
\text { nephrotoxicity) }\end{array}$ & $\begin{array}{l}\text { Full term normal vaginal } \\
\text { delivery at } 38 \text { weeks of gestation }\end{array}$ \\
\hline
\end{tabular}


Table 2. (Continued)

\begin{tabular}{|c|c|c|c|c|c|c|}
\hline Author-year & Country & $\begin{array}{l}\text { Time of detection/ } \\
\text { description }\end{array}$ & $\begin{array}{l}\text { Number of } \\
\text { pregnant } \\
\text { woman/ } \\
\text { women }\end{array}$ & Trimester & $\begin{array}{l}\text { Description of treatment in } \\
\text { pregnancy }\end{array}$ & Pregnancy outcome \\
\hline Kumar-2015 [105] & India & $\begin{array}{l}\text { Retrospectively } \\
\text { identified (After } 5 \\
\text { months of delivery) }\end{array}$ & 1 & $3^{\text {rd }}$ & Untreated & Normal vaginal birth \\
\hline $\begin{array}{l}\text { AlmeidaSilva-2015 } \\
\text { [50] }\end{array}$ & Brazil & During pregnancy & 1 & $1^{\text {st }}$ & L-AmB & Foetal death \\
\hline Basher -2017 [65] & Bangladesh & During pregnancy & 5 & No information & $\begin{array}{l}\text { One untreated; One was treated } \\
\text { with L-AmB }\end{array}$ & Untreated mother died \\
\hline $\begin{array}{l}\text { Kimutai-2017 [58] } \\
\text { (personal } \\
\text { communication with } \\
\text { Dr Alves) }\end{array}$ & East Africa & During pregnancy & 8 & No information & $\mathrm{SSG}+\mathrm{PM}$ & Spontaneous abortion $(\mathrm{n}=1)$ \\
\hline $\begin{array}{l}\text { Panagopoulos-2017 } \\
{[106]}\end{array}$ & Greece & During pregnancy & 1 & $3^{\text {rd }}$ & $\begin{array}{l}\mathrm{L}-\mathrm{AmB}(3 \mathrm{mg} / \mathrm{kg} / \text { day for } 5 \text { days } \\
\text { and on days } 14 \text { and } 21)\end{array}$ & Normal term birth \\
\hline Adam-2018 [107] & Sudan & During pregnancy & 45 & Mostly $3^{\text {rd }}$ & No information & $\begin{array}{l}8 \text { maternal death ( } 6 \text { in prenatal } \\
\text { and } 2 \text { in postnatal); } 37 \text { survived; } \\
30 \text { were full term; } 6 \text { pre-term } \\
\text { birth; } 2 \text { spontaneous abortion; } 1 \\
\text { stillbirth }\end{array}$ \\
\hline $\begin{array}{l}\text { Goyal-2018 [108] } \\
\text { (personal } \\
\text { communication With } \\
\text { Dr Alves) }\end{array}$ & India & During pregnancy & 2 & No information & $\begin{array}{l}\text { Single dose AmBisome (10 mg/ } \\
\mathrm{kg})\end{array}$ & No complications \\
\hline Russo-2018 [109] & Italy & $\begin{array}{l}\text { Retrospectively } \\
\text { identified }\end{array}$ & 1 & $\begin{array}{l}\text { Not applicable } \\
\text { (retrospective) }\end{array}$ & No information & Vertical transmission \\
\hline Cunha-2019 [110] & Brazil & During pregnancy & 1 & $3^{\text {rd }}$ & L-AmB (3 mg/kg for 7 days) & $\begin{array}{l}\text { Normal term birth without } \\
\text { complications }\end{array}$ \\
\hline Argy-2019 [53] & Brazil & During pregnancy & 1 & $3^{\text {rd }}$ & L-AmB & Vertical transmission at birth \\
\hline Parise-2019 [111] & France & $\begin{array}{l}\text { Retrospectively } \\
\text { identified }\end{array}$ & 1 & $\begin{array}{l}\text { Not applicable } \\
\text { (retrospective) }\end{array}$ & Untreated during pregnancy & Maternal death \\
\hline $\begin{array}{l}\text { Pekelharing-2020 } \\
\text { [11] }\end{array}$ & $\begin{array}{l}\text { South } \\
\text { Sudan }\end{array}$ & During pregnancy & 87 & $\begin{array}{l}26.8 \% \text { in } 1^{\text {st }} \\
35.1 \% \text { in } 2^{\text {nd }} \\
38.1 \% \text { in } 3^{\text {rd }}\end{array}$ & $\mathrm{L}-\mathrm{AmB}(30 \mathrm{mg} / \mathrm{kg}$ in 6 doses $)$ & $\begin{array}{l}1 \text { maternal death; } 16 \text { adverse } \\
\text { pregnancy outcomes }\end{array}$ \\
\hline $\begin{array}{l}\text { Pekelharing-2020 } \\
\text { [11] }\end{array}$ & $\begin{array}{l}\text { South } \\
\text { Sudan }\end{array}$ & $\begin{array}{l}\text { Retrospectively } \\
\text { identified (two weeks } \\
\text { post-partum) }\end{array}$ & 26 & $\begin{array}{l}\text { Not applicable } \\
\text { (retrospective) }\end{array}$ & $\mathrm{L}-\mathrm{AmB}(30 \mathrm{mg} / \mathrm{kg}$ in 6 doses $)$ & $\begin{array}{l}1 \text { maternal death; } 13 \text { adverse } \\
\text { pregnancy outcomes }\end{array}$ \\
\hline
\end{tabular}

L- AmB = Liposomal amphotericin B; PA = pentavalent antimony; SSG = sodium stibogluconate; SAG = Sodium antimony gluconate; IV = intravenous; $\mathrm{IM}=$ intramuscular; PM = Paromomycin; Mueller-2007 [87] was identified after completion of the review and not included in this table - the study described 5 pregnant women treated with AmBisome: six doses of $2.5-8.2 \mathrm{mg} / \mathrm{kg}$ on days 1,2, 3, 5, 10, 15 with no pregnancy outcomes not reported

treatment status was not clear in the remaining 49 (12.3\%) (Table 2). The outcomes among 3 untreated cases and 346 who received a treatment during pregnancy is presented next.

Liposomal amphotericin $B(\mathbf{n}=202 ; \mathbf{1 9 9 4 - 2 0 2 0})$. Of the 202 pregnant women treated with Liposomal Amphotericin B, 124 (61.4\%) were from Africa (2006-2020), 59 (29.2\%) were from the Indian sub-continent (2010-2018), 13 (6.4\%) were from Europe (2010-2018), and 6 (3.0\%) were from South America (2005-2019).

Treatment was administered in $5(2.5 \%)$ cases in the first trimester, $8(4.0 \%)$ in the second trimester, $9(4.5 \%)$ in the third trimester, while the time in pregnancy was not clear in 180 (89.1\%). Survival status was not reported or was unclear in 26 (12.9\%) pregnant women and 

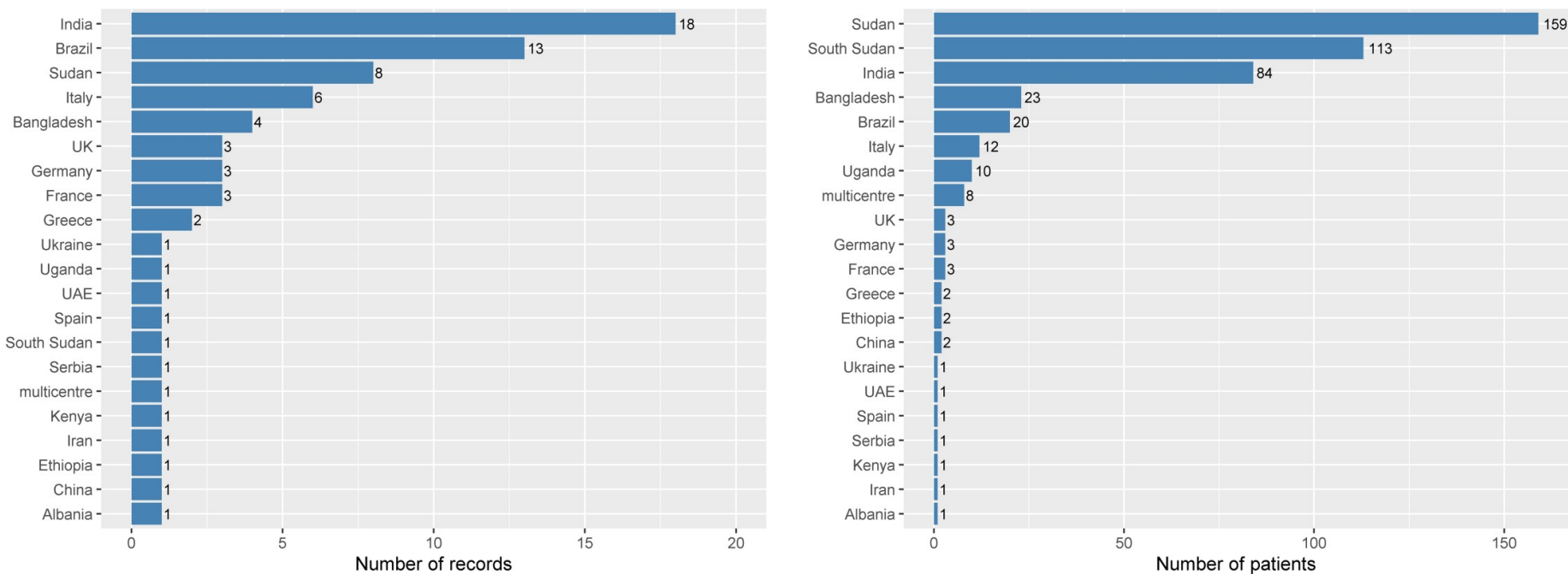

Fig 2. Number of records and patients by country of origin.

https://doi.org/10.1371/journal.pntd.0009650.g002

from the remaining 176 , a total of four $(2.3 \%, 4 / 176)$ maternal deaths were reported. There were five (2.8\%) miscarriages, $1(0.6 \%)$ stillbirth, and $1(0.6 \%)$ premature birth (Table 3$)$. One foetal death was reported from a pregnant woman in her first trimester from South America [50].

Three cases of vertical transmission were identified [51-53] (Tables 2 and 4). The first one was detected at 11 months after birth. The pregnant woman was admitted to a hospital few days before labour and was administered L-AmB at a daily dose of $4 \mathrm{mg} / \mathrm{kg}$ on 6 consecutive days and repeated doses at days 14 and 21 [51]. The treatment status on the baby was not available. For the

Table 3. Reported unfavourable maternal outcomes after liposomal amphotericin B and antimony treatment among those who were treated during pregnancy.

\begin{tabular}{|c|c|c|c|c|c|}
\hline & Africa & Europe & South America & Southern Asia & Total \\
\hline \multicolumn{6}{|l|}{ Liposomal amphotericin B } \\
\hline Total treated & 124 & 13 & 6 & 59 & 202 \\
\hline \multicolumn{6}{|l|}{ Outcomes (reported in 176; unclear in 26) } \\
\hline Abortion/spontaneous abortion & - & - & - & - & - \\
\hline Miscarriage & 5 & - & - & - & 5 \\
\hline Premature birth & 1 & - & - & - & 1 \\
\hline Stillbirth & - & - & - & 1 & 1 \\
\hline Foetal death & - & - & 1 & - & 1 \\
\hline Maternal death & 3 & - & - & 1 & 4 \\
\hline \multicolumn{6}{|l|}{ Pentavalent antimony } \\
\hline Total treated & 70 & 2 & 2 & 19 & 93 \\
\hline \multicolumn{6}{|l|}{ Outcomes (reported in 88; unclear in 5) } \\
\hline Abortion/spontaneous abortion & 13 & - & - & 11 & 24 \\
\hline Miscarriage & 2 & - & - & - & 2 \\
\hline Premature birth & 2 & - & $1^{\mathrm{a}}$ & - & 3 \\
\hline Stillbirth & - & - & - & - & - \\
\hline Foetal death & 2 & - & $1^{\mathrm{a}}$ & - & 3 \\
\hline Maternal death & 4 & & & & 4 \\
\hline Other (required splenectomy after delivery) & - & - & - & 1 & 1 \\
\hline
\end{tabular}

${ }^{a}$ Indicates the same patient (the baby was born prematurely and died a day after birth) 
Table 4. Details of confirmed, probable, or suspected cases of vertically transmitted visceral leishmaniasis.

\begin{tabular}{|c|c|c|}
\hline Study ${ }^{a}$ & Location & Case description \\
\hline Low -1926 [19] & UK & $\begin{array}{l}\text { A retrospective description of a child born to a mother who contracted the disease during pregnancy while residing in India } \\
\text { and had given birth in the UK. }\end{array}$ \\
\hline Hindle-1928 [66] & China & $\begin{array}{l}\text { A four months old baby whose spleen puncture confirmed the presence of Leishmania parasites. } \\
\text { "The main interest of this case lies in the fact that it could not possibly have been exposed to the bites of sandflies, as their } \\
\text { season ended approximately two months before the child was born. Although the mother showed no obvious signs of disease it } \\
\text { is difficult of explanation except on the hypothesis of congenital transmission. Low and Cooke (1926) recorded a case of Indian } \\
\text { Kala-azar in a child born in England, and there can be no doubt that in this patient the infection was derived from the mother } \\
\text { who was also infected" }\end{array}$ \\
\hline Hindle-1928 [66] & China & $\begin{array}{l}\text { "Dr Marshall Hertig kindly informed me of a similar case at Hsii-Chowfu in which the patient, a five months old child, was } \\
\text { successfully treated for Kala-azar at the local mission hospital. This infant also, from the date of its birth, could never have been } \\
\text { exposed to the bites of sandflies" }\end{array}$ \\
\hline Banerji-1955 [56] & India & $\begin{array}{l}\text { Mother contracted kala-azar in the fifth month of pregnancy and suspected vertical transmission occurred when the child was } 6 \\
\text { months old. }\end{array}$ \\
\hline Blanc-1984 [68] & France & $\begin{array}{l}\text { Mother with a subclinical infection during pregnancy with the disease detected within a month after delivery. } \\
\text { The child had a confirmed VL and was the first case reported in the hospital. The child never left the hospital and never came in } \\
\text { contact of dogs thus suggesting that vertical transmission was the likely mode of transmission. }\end{array}$ \\
\hline Mittal-1987 [62] & India & $\begin{array}{l}\text { "An 11-months-old male infant admitted with symptoms that were later confirmed as VL. The baby's mother had also suffered } \\
\text { from kala-azar while carrying this child. As the baby and his mother did not leave New Delhi, India, where the case was related, } \\
\text { either during or after the delivery and the vector found in New Delhi was not competent to transmit leishmaniasis, the infant } \\
\text { could not have been infected by the bite of a sandfly. It therefore seems most likely that he was congenitally exposed to kala- } \\
\text { azar." }\end{array}$ \\
\hline Nyakundi-1988 [70 $]^{\mathrm{b}}$ & Kenya & $\begin{array}{l}\text { "We recently treated a } 4 \text { months old male infant born prematurely on } 18 \text { June 1986, after } 6 \text { months gestation to a then febrile } \\
\text { (para 6+3) mother diagnosed as having had kala-azar during pregnancy. Mother and infant were admitted to the Clinical } \\
\text { Research Centre. Kenya Medical Research Institute, on } 20 \text { October-1986; when kala-azar was confirmed in the mother. This } \\
\text { infant with congenital kala-azar was only the fourth and youngest patient with this disease ever reported in the world medical } \\
\text { literature. The mode of infection in the baby could be (a) direct transmission from mother to offspring, (b) acquired in hospital, } \\
\text { (c) acquired at the time of birth from perineal haemorrhages with swallowing of maternal blood or secretions or through the } \\
\text { cord or skin abrasions, or (d) acquired congenitally from the mother through the placenta. Only the last of these possible modes } \\
\text { of transmission is likely in view of the poor health of the infant from the 6th day of life, the mother's bad obstetric history, the } \\
\text { hospital's high altitude which makes it unsuitable for sandfly transmission, and because the period that elapsed from birth to the } \\
\text { appearance of symptoms was compatible with a congenital infection." }\end{array}$ \\
\hline Yadav-1989 [71] & India & $\begin{array}{l}\text { An 11-months male infant was admitted with kala-azar. The mother suffered from the disease during pregnancy. The mother } \\
\text { from Bihar migrated to Delhi during first trimester. She showed signs of disease during sixth month of pregnancy. The most } \\
\text { likely mode of infection was in utero transmission of the disease. }\end{array}$ \\
\hline Eltoum-1992 [18] & Sudan & $\begin{array}{l}\text { During an epidemic of visceral leishmaniasis in the Sudan, two cases of congenital kala-azar were seen. } \\
\text { The first child, whose mother had contracted kala-azar in southern Sudan, was born in Khartoum, where no transmission of } \\
\text { leishmaniasis is currently occurring. At seven months, the child had fever, lymphadenopathy, and hepatosplenomegaly; } \\
\text { leishmania parasites were detected in the bone marrow. The child died and an autopsy showed leishmania parasites in all tissues } \\
\text { including the lungs, kidneys, and thymus. } \\
\text { In the second case, parasites were found in the placenta of a five-months-old foetus. }\end{array}$ \\
\hline Elamin -1992 [17] & Sudan & $\begin{array}{l}\text { A case of visceral leishmaniasis was described in a 6-weeks-old infant from southern Sudan who most likely got the infection } \\
\text { through transplacental transmission. This was described as the first reported case of congenital kala-azar in Africa and the } \\
\text { seventh in the global medical literature }\end{array}$ \\
\hline Sharma-1996 [77] & India & $\begin{array}{l}\text { "Thus, in all possibility, it was a case of congenital kala-azar acquired transplacental by the baby from a mother having } \\
\text { subclinical kala-azar."The infection was possibly active when the child was } 4 \text { months of age and it was detected when the child } \\
\text { was } 18 \text { months. }\end{array}$ \\
\hline Meinecke-1999 [79] & Germany & $\begin{array}{l}\text { A case of VL was reported in a child who had never left Germany. A nonvector transmission was suspected and therefore } \\
\text { household contacts were examined. His mother was the only one who had a positive antibody titre against Leishmania } \\
\text { donovani complex. She had travelled several times to endemic Mediterranean areas (Portugal, Malta, and Corse) before giving } \\
\text { birth to the boy. But she had never been symptomatic for visceral leishmaniasis. Her bone marrow, spleen, and liver biopsy } \\
\text { results were within normal limits. }\end{array}$ \\
\hline Boehme-2006 [86] & Germany & $\begin{array}{l}\text { A case of VL was reported in a German infant, who never had been to a VL endemic area. Most likely, the parasite was } \\
\text { congenitally transmitted from the asymptomatic mother to her child. }\end{array}$ \\
\hline Papageorgiou-2010 [51] & Greece & $\begin{array}{l}\text { This article reports the first case of congenital VL in Greece. The mother of the infant was hospitalised a few days before labour } \\
\text { because of anaemia and hepatosplenomegaly, and titres for Leishmania antibodies were positive. A bone marrow aspirate } \\
\text { showed no evidence of malignancy, except from a slight decrease of myelopoiesis, erythropoiesis and thrombopoiesis. However, } \\
\text { the promastigote form of Leishmania was found, and therefore, diagnosis of leishmaniasis was confirmed. }\end{array}$ \\
\hline Haque-2010 [91] & Bangladesh & The article is the first report of vertical transmission of VL in Bangladesh. \\
\hline
\end{tabular}


Table 4. (Continued)

\begin{tabular}{|c|c|c|}
\hline Study ${ }^{\mathbf{a}}$ & Location & Case description \\
\hline Zinchuk -2010 [52] & Ukraine & $\begin{array}{l}\text { An } 8 \text {-months-old boy was diagnosed with visceral leishmaniasis in Ukraine, a non-endemic area. His mother had been treated } \\
\text { for visceral leishmaniasis at } 28-32 \text { weeks gestation whilst working in Alicante, Spain and delivered her infant at } 38 \text { weeks } \\
\text { gestation by elective caesarean section in Ukraine. The authors presumed that the infant's infection was as a result of vertical } \\
\text { transmission. }\end{array}$ \\
\hline Pilaca-2011 [93] & Albania & $\begin{array}{l}\text { Leishmania amastigotes were detected in bone marrow biopsy of the mother. Two days later, premature birth was stimulated. } \\
\text { After } 2-3 \text { months of the birth the baby was not well. After admitted to hospital, baby resulted positive for VL. He was treated } \\
\text { with Glucantime and was cured after a scheme of two } 14 \text {-day cycles with good outcome. }\end{array}$ \\
\hline Damodaran-2012 [94] & UK & $\begin{array}{l}\text { "A } 15 \text { months-old-girl, family from East Timor, referred from primary care with weight-loss and a non-healing skin ulcer. She } \\
\text { appeared undernourished with pallor, pyrexia and hepatosplenomegaly. FBC showed pancytopenia. Bone marrow examination } \\
\text { confirmed Leishmaniasis. Her mother had intrapartum Leishmaniasis. The child was born in United Kingdom with no history } \\
\text { of foreign travel and responded well to treatment with AmBisome" }\end{array}$ \\
\hline $\begin{array}{l}\text { Mescouto-Borges-2013 } \\
{[96]}\end{array}$ & Brazil & $\begin{array}{l}\text { Two cases of congenitally transmitted visceral Leishmaniasis was reported. Both mothers had developed symptoms of VL } \\
\text { during pregnancy. The diagnosis was made by visual examination of Leishmania parasites in bone marrow aspirates of the } \\
\text { mothers and by detecting parasite DNA in bone marrow samples of the new-born children using polymerase chain reaction. }\end{array}$ \\
\hline Bode-2014 [100] & Germany & $\begin{array}{l}\text { "One infant girl (P8) had only been in an endemic area (Spain) in utero. Vertical transmission resulting in congenital visceral } \\
\text { leishmaniasis must be assumed, as the mother, who remained clinically asymptomatic, was serologically positive. Diagnosis of } \\
\text { visceral leishmaniasis was delayed for more than } 3 \text { weeks". } \\
\text { The girl had never been abroad after birth and the mother had positive Leishmania serology after a trip to Spain during } \\
\text { pregnancy. }\end{array}$ \\
\hline Kumar-2015 [105] & India & $\begin{array}{l}\text { The authors presumed that the infant's infection was a result of vertical transmission. The authors presumed that the mother } \\
\text { might have had subclinical infection and transmitted the disease to the offspring. }\end{array}$ \\
\hline Basher -2017 [65] & Bangladesh & $\begin{array}{l}\text { The authors described a mother who had died before VL treatment began. The mother died after giving birth to a foetus. Foetal } \\
\text { part placenta was collected and upon examination using PCR, the sample tested positive for LD body. The authors reported that } \\
\text { Kala-azar in the mother may have been the cause of the foetal wastage. }\end{array}$ \\
\hline Russo-2018 [109] & Italy & The authors reported a vertically transmitted Leishmaniasis in a 6-months old girl whose parents were from Southern Italy. \\
\hline Argy-2019 [53] & France & $\begin{array}{l}\text { A case of transplacentally transmitted VL from the HIV-positive pregnant mother to the child. Few intracellular Leishmania } \\
\text { amastigotes were found during the microscopic examination of the placenta confirmed by positive PCR results. }\end{array}$ \\
\hline
\end{tabular}

$\mathrm{VL}=$ Visceral leishmaniasis PCR $=$ Polymerase chain reaction

${ }^{\text {a }}$ An article from Sudan (Adam 2009 [12]) described a case of a 2 months baby with parasites detected in lymph node. The article did not mention whether this could be a case of vertical transmission.

${ }^{b}$ In Nyakundi-1988[70], three cases of vertically transmitted VL in clinical literature were identified: Low and Cooke-1926[19]; Banerji-1955[56] and Napier-1946[112]. The first two reports are included in this table whereas we have decided not to include the last report as a case of vertical transmission as the original article could not be retrieved and case details couldn't be verified. The following description appears in Napier-1946 [112]: "Even in India kala-azar occurs among infants; we reported a case of an infant of less than eight months with well-developed kala-azar of about four months' duration". While it is clear that VL was identified when the infant was four months old, there is no further description of the case [112]. The brief description in Napier-1946[112] matches an earlier publication (Napier and Das Gupta-1928 [113]) in which the plausibility of vertical transmission was ruled out: "As the mother showed no sign of the disease at all it is extremely unlikely that the child was suffering from the disease at birth."

https://doi.org/10.1371/journal.pntd.0009650.t004

second case, vertical transmission was suspected at 8 months after birth. During weeks $31-32$ of pregnancy, the pregnant woman was treated with a full course of L-AmB $(3 \mathrm{mg} / \mathrm{kg}$ on days $1-5$ followed by a single dose of $3 \mathrm{mg} / \mathrm{kg}$ on day 10). Delivery by an elective C-section occurred at 38 weeks of gestation. Upon detection of the Leishmania parasites in the bone marrow, the baby was treated with sodium stibogluconate at an intravenous dose of $20 \mathrm{mg} / \mathrm{kg}$ for 20 days and discharged after 30 days [52]. The third one was detected in a baby born to a mother with HIV coinfection immediately after vaginal birth [53]. Leishmania amastigotes were found during the microscopic examination of the placenta, and diagnosis was confirmed by a polymerase chain reaction. The baby was administered intravenous L-AmB at dosage of $5 \mathrm{mg} / \mathrm{kg}$ per day from days 1 to 4 followed by a weekly injection of $5 \mathrm{mg} / \mathrm{kg}$ and recovered successfully. The mother had received L-AmB 15 weeks after her last menstrual period at a total dose of $40 \mathrm{mg} / \mathrm{kg}$, followed by secondary prophylaxis with a dose of $5 \mathrm{mg} / \mathrm{kg}$ every 15 days until delivery. 
Pentavalent antimony $(\mathbf{n}=\mathbf{9 3} ; \mathbf{1 9 2 6}-\mathbf{2 0 1 0})$. There were 93 pregnant women who were treated with pentavalent antimony, of whom 70 (75.3\%) were from Africa (1984-2009), 19 (20.4\%) from Asia (1955-2010), 2 (2.2\%) from Europe (1926-1995) and 2 (2.2\%) from South America (1994-2003) with majority of the cases treated between 2000-2010 (82 of 93 cases). Treatment was administered to 20 (21.5\%) pregnant women in the first trimester, 46 (49.5\%) in the second, $22(23.7 \%)$ in the third, while the time in pregnancy was not clear in $5(5.4 \%)$.

Survival status was available for $88(94.6 \%)$ pregnant women of whom $4(4.5 \%, 4 / 88)$ died due to hepatic encephalopathy [12]. There were 24 (27.3\%) abortions (or spontaneous abortions) of which 13 were among African patients and 11 among patients from Asia (Table 3). There were $2(2.2 \%)$ miscarriages, $2(2.2 \%)$ pre-term births, and $1(1.1 \%)$ required splenectomy after delivery due to poor recovery and due to persistence of parasitaemia in the spleen sample (Table 3) [54]. There were 3 foetal deaths reported; one of the babies died due to myelomeningocele 3 hours after birth [12], another died a day after being born [55], and another died due to VL at 2 months [12]. Further details are presented in Table 3.

There were 3 cases of vertical transmission identified $[18,19,56]$, detected at 6,7 , and 12 months after birth. The first case (reported in 1926 in the UK) was born to a woman who acquired VL during her third trimester and was treated with urea stibamine [19]. The baby had a normal birth and vertical transmission was detected at 12 months after birth. Upon detection, the baby was treated with antimony and made a recovery. The second case (reported in 1955 in India) was born to a woman in whom the disease was identified during the second trimester and was treated with urea stibamine [56]. Vertical transmission was detected at 6 months; the baby was treated with antimony and was cured after one recurrence. The third case (reported in Sudan in 1922) was born to a woman in whom the disease was identified during the second trimester and was treated with sodium stibogluconate for 30 days [18]. At birth, the baby had signs of intrauterine growth retardation. The baby was diagnosed with VL at 7 months and died two days later (Table 4).

Amphotericin B deoxycholate $(\mathbf{n}=\mathbf{2 0}$; 1993-2015). Of the 20 pregnant women treated with amphotericin B deoxycholate, 17 (85.0\%) were from Asia (1993-2015) and 3 (15.0\%) from South America (2003-2008). There were 3 (15.0\%) pregnant women in their first trimester, 6 $(30.0 \%)$ in the second, $3(15.0 \%)$ in the third, and the trimester was not clear in $8(40.0 \%)$. There was one (5.0\%) maternal death after 7 days of treatment due to haemorrhagic complications occurring after delivery; the pregnant woman was in $28.7 \pm 7.8$ weeks of pregnancy [57]. The remaining 19 pregnant women were discharged alive. The delivery of babies were described as normal for 18 pregnant women, haemorrhagic complication occurred in one after delivery (as described earlier) [57], and the information was not reported on 1 (Table 2 and S2 Data). There was no reports of vertical transmission of the disease in 12 (60.0\%) babies in whom the information was reported (11 were followed-up for 12-months and the status was reported at birth for 1 baby). Further details are presented supplemental file (S2 Data).

Pentavalent antimony plus paromomycin (aminosidine) $(n=11 ; 1993-2017)$. Eleven pregnant women (all from Africa) were treated with sodium stibogluconate plus aminosidine (paromomycin) [27, 58]. Information regarding trimester, maternal survival status, or vertical transmission were not available (see S2 Data). One spontaneous abortion was reported [58].

Liposomal amphotericin B plus pentavalent antimony $(n=4$; 2006). Four pregnant women were treated with the combination regimen in a study from Sudan published in 2006 [59], of whom two were in their second trimester and two in their third. L-AmB was administered at 3-7 mg/kg/day on days 1, 6, 11 and 16 (or on days 1, 2, 3, 4, 10 and 15), followed by a $20 \mathrm{mg} / \mathrm{kg}$ dose of sodium stibogluconate intramuscularly once daily for 30 days. All four pregnant women were discharged alive. At discharge, one pregnant woman delivered a healthy baby and the remaining three were still pregnant-no follow-up data was reported. 
Paromomycin (aminosidine) $(\mathbf{n}=3 ; \mathbf{2 0 1 1})$. Three pregnant women were treated with paromomycin in a study from India [60]. The drug regimen was administered at $11 \mathrm{mg} / \mathrm{kg} /$ day (base) by deep gluteal intramuscular injection once daily for 21 consecutive days [60]. All three babies were described as being healthy at birth.

Trimothoprim, sulphadiazine and tinidazole $(\mathbf{n}=1 ; \mathbf{1 9 9 4 )})$. One study from India treated a pregnant woman in the first trimester with the combination of Trimothoprim, sulphadiazine and tinidazole [61]. There were no reports of side effects and the pregnant woman delivered a healthy baby at term. The mother remained well at one year after completion of treatment with no further information available on the infant.

Unclear drug substance $(n=12 ; 1987-2009)$. Three publications reported treating pregnant women with VL in whom either the drug name was not specified or the allocated drug group (in multi-arm study) could not be identified. Two publications described a case each without reporting the name of the drug administered [17, 62]. In the third report, the number of pregnant women $(\mathrm{n}=10)$ allocated to each drug arm (pentavalent antimony or amphotericin $\mathrm{B}$ deoxycholate) was not clear [63]. Two (16.7\%) of the pregnant women were in their third trimester and the status was unknown for the remaining $10(83.3 \%)$. There were two (16.7\%) maternal deaths [63] and two cases of vertical transmission $[17,62]$. The first one was identified at 8 months after birth and another at 6 weeks after birth (the baby died after 3 days). Both babies were administered treatment upon detection of VL (See S2 Data for further details).

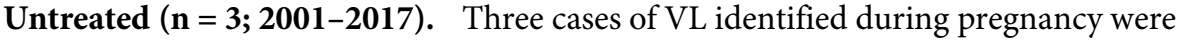
untreated $[25,64,65]$. Treatment was deferred until after delivery due to safety concerns in one report in a pregnant women in third trimester [25]; there were signs of intra-uterine growth retardation requiring an emergency $\mathrm{C}$-section, and both mother and the child were alive. The second pregnant woman in third trimester was not treated due to lack of adequate hospital resources [64]; the baby born to the mother died after 2 months due to malnutrition with no evidence of vertical transmission. In the third case, VL was diagnosed nearer the delivery time before treatment could be administered but the mother died after giving birth and before treatment could be administered [65]; the baby also died and foetal part placenta examination revealed presence of Leishman Donovan bodies by polymerase chain reaction indicating vertical transmission.

\section{Confirmed/probable/suspected vertical transmission}

We identified a total of 26 cases of confirmed, probable or suspected cases of vertical transmission (Table 4). The median time to detect vertically transmitted VL was 6 months (range: $0-18$ months). Eleven babies were born to women in whom the disease status was confirmed during their pregnancy; three of the mothers were treated with L-AmB, three with PA, the drug name was not clear in two, a mother was untreated and the treatment status was not clear in the remaining two (Table 4). Histopathological examination of the placenta confirmed the vertical transmission of the disease in two cases $[18,65]$ and this was not reported for the remaining cases. Treatment status was described in 18 children, of whom 11 received pentavalent antimony, 6 received L-AmB and 1 received amphotericin $\mathrm{b}$ deoxycholate. Two of the children died; one had received pentavalent antimony and the drug name was not clear in the other (See S2 Data for further details).

\section{Risk of bias assessment}

Of the 71 case reports/case series, $49(69.0 \%)$ were judged to be at a high risk of bias for patient selection and the remaining $22(31.0 \%)$ were at low risk of bias. For the ascertainment of exposure status, 6 (8.5\%) were at high risk of bias, 17 (23.9\%) were at moderate risk, 41 (57.7\%) at 
low risk, and the risk of bias status was not clear in 7 (9.9\%) studies. For the adequacy of follow-up, four (5.6\%) studies were considered to be at moderate-high risk of bias, 37 (52.1\%) at low risk of bias, 23 (32.4\%) were retrospective studies, and the risk of bias was unclear in 7 (9.9\%). For the completeness of reporting domain, 5 (7.0\%) studies were at high risk of incomplete reporting, 20 (28.2\%) at moderate risk of bias, and 46 (64.8\%) were at low risk of bias (See S1 Table). One retrospective cohort study with a comparative group of non-pregnant patient group was considered of high quality.

\section{Discussion}

The occurrence and effects of VL during pregnancy is under-researched and poorly understood as evidenced by having identified only 72 publications describing a total of 451 cases of $\mathrm{VL}$ in pregnancy in the past 94 years.

The small case volume reported in the literature could have several explanations. In the first place, there is an apparent imbalance in caseloads with predominance of the disease among males; ascribed to biological or behavioural causes [10, 13, 73, 114-116]. Pentavalent antimony was the first line therapy before the development of Liposomal amphotericin B (L-AmB)-this might have traditionally dissuaded physicians from treating VL during pregnancy and leaning towards postponing the treatment until after delivery unless treatment is absolutely warranted [25]. However, this situation might have changed recently as L-AmB has no contraindication during pregnancy and is the treatment of choice adopted in national guidelines (Table 1). It has also been postulated that early pregnancies are missed due to spontaneous abortion caused by VL [73]. Women with childbearing potential or those who are already pregnant are systematically excluded from VL clinical studies and only a third of the patients enrolled in clinical trials are females [10]. It is clear that the likely caseload of VL in pregnancy is much bigger than what can be estimated from available reports. For example, during Jan 2016-Jul 2019 in Lankien, Jonglei state, South Sudan, out of 4,448 cases of VL diagnosed, 824 (18.5\%) occurred in females aged $15-44$ years and $110(13 \%, 110 / 824)$ of them were pregnant $(2.5 \%$ of all cases) (Personal communication with Dr. Ritmeijer).

There was also geographical disparity in the treatment regimens used. Among the cases identified during pregnancy, just over two-thirds of the patients received amphotericin B regimens (any formulations or in combination with another drug) in studies conducted in Africa compared to more than $75 \%$ of the patients from Asia. This could reflect either heterogeneity in treatment practices or the differential parasite susceptibility against drug regimens in the regions.

Among 346 cases in whom VL was diagnosed during pregnancy and received a treatment, there were a total of 11 (3.2\%) maternal deaths reported; four $(4.5 \%, 4 / 88)$ occurred in those treated with pentavalent antimony-based regimens, 4 (4/176; $2.3 \%)$ among those treated with $\mathrm{L}-\mathrm{AmB}, 1(1 / 20,5.0 \%)$ with amphotericin b deoxycholate, and the drug name used for the treatment was not clear in $2(16.7 \%, 2 / 12)$ cases. Abortion following PA regimen was observed in just over a quarter of the pregnant women $(24 / 88,27.3 \%)$ of whom 13 were from Africa and 11 were from Indian sub-continent. Following the $\mathrm{L}-\mathrm{AmB}$ regimen, there were a total of 5 $(2.8 \%)$ miscarriages and $1(0.6 \%)$ foetal death and no cases of abortion were reported (Table 3). Taken together, these results support the use of liposomal amphotericin B for the treatment of VL during pregnancy.

Our review identified 26 confirmed/suspected/possible cases of vertically transmitted VL with a median time of detection of 6 months (range: 0-18 months); 12 (46\%) cases were identified after 6 months of birth. This suggests that children born to mothers with VL during pregnancy may require a longer post-treatment follow-up than the standard 6-months follow-up 
duration among non-pregnant patients to identify cases of vertical transmission. The underlying mechanism of the onset of clinical leishmaniasis among neonates and infants born to a successfully-treated mother during pregnancy is currently not clear; it has been ascribed to imbalances in immune-mechanism modulated by $\mathrm{T}$ cell responses (Th1/Th2) [24] or by parasites entering a state of dormancy in the lymph nodes [81].

Our review has identified limitations in reporting of VL in pregnancy. Complete information was often not available on treatment administered or on efficacy and safety outcomes for the mother and baby. For 12\% (54/451) of the pregnant women, it could not be ascertained whether they had received any treatment or not. Just over two-third of the studies were considered to be a high risk of bias for patient selection while a nearly a third of the studies were at moderate to high risk of bias for ascertainment of exposure domain as VL diagnosis was purely based on clinical signs and symptoms or on serological methods (S1 Table). Overall, the large number of case reports/case series included in this review provides an explanation for such high risk of bias and likely indicates that the reported evidence would be regarded as low quality. This review also suggested that existing practices for management of VL in pregnancy is guided by limited evidence generated from case reports and small case series ( 71 of the 72 studies were case-reports/case series). High quality studies (such as Pekelharing-2020 [11]) is warranted for generation of a robust evidence regarding safety and efficacy of antileishmanial agents during pregnancy. There was also a lack of standardised reporting as information was missing on several critical parameters such as trimester status, time of detection of VL, and therapeutic outcomes for the mother and the child. Taken together, these findings highlight the need to improve and harmonise the reporting of VL in pregnant women. We have outlined a minimum checklist of items that might be useful for reporting purposes (Box 1).

\begin{tabular}{l|l}
\hline Domain & Checklist Item \\
\hline Maternal history & Parity \\
\hline & Gravidity \\
\hline & Maternal history of the disease \\
\hline & History of travelling to endemic regions \\
\hline Maternal characteristics & Any previous treatment of the disease \\
\hline & Comorbidities (HIV, malaria, TB etc.) \\
\hline & Age \\
\hline & Weight \\
\hline Maternal clinical signs and symptoms & Nutritional status \\
\hline & Trimester \\
\hline & Gestational age \\
\hline & Haemoglobin on admission \\
\hline Diagnostics & Hepatomegaly status \\
\hline & Splenomegaly \\
\hline & Diagnostic method used (PCR, ELISA, rk39DAT, IFA) \\
\hline & Sample analysed (blood, bone marrow aspirates, splenic aspirates etc) \\
\hline & Method of confirmation of disease status \\
\hline & Parasite species (L. donovani, L. infantum) \\
\hline & Method used for estimating gestational age (ultrasonography etc) \\
\hline & Dose, duration, frequency including mode of administration \\
\hline &
\end{tabular}


Table 4. (Continued)

\begin{tabular}{l|l}
\hline & $\begin{array}{l}\text { Concomitant medication status (antipyretics, antimalarial etc) } \\
\text { Need for blood transfusion before or during treatment }\end{array}$ \\
\hline Delivery characteristics & Mode of delivery (C-section, natural) \\
\hline & Trauma during delivery \\
\hline & APGAR score \\
\hline & Examination of placenta \\
\hline & Birth status (still birth, abortion, healthy birth) \\
\hline & Any birth-related complications \\
\hline & Clinical response (initial cure/default/non-response/death) \\
\hline & Adverse events \\
\hline & Fetal outcome status: abortion/stillbirth/premature birth/healthy birth \\
\hline & Follow-up data on babies \\
\hline
\end{tabular}

PCR = Polymerase chain reaction; ELISA = enzyme-linked immunosorbent assay; IFA = Immunofluorescence assay https://doi.org/10.1371/journal.pntd.0009650.t005

In addition to the articles presented in this review, data from women who become pregnant after completion of therapy but within the follow-up period enrolled in trials might provide further evidence on drug safety, especially on the reproductive consequences of the treatment (Table 5). The recently proposed safe ethical framework for the recruitment of women susceptible to and becoming pregnant is an important development towards filling the existing knowledge gap [28]. Like for many neglected tropical diseases, there is currently an absence of a comprehensive pregnancy-specific registry for exposures to antileishmanials, with the exception of the one dedicated for miltefosine [117]. Therefore, creating an open registry where all these cases are indexed and continually updated would help in better characterisation of the safety aspects of the drugs. Finally, the Infectious Diseases Data Observatory (IDDO) data platform, that is currently standardising individual participant data from several VL clinical studies, offers a unique resource to explore host, parasite, and drug dynamics affecting the safety and efficacy in pregnant populations [118].

Table 5. Description of patients enrolled in clinical trials who became pregnant after completion of treatment.

\begin{tabular}{|c|c|c|c|}
\hline Study & $\begin{array}{l}\text { Number of } \\
\text { patients }\end{array}$ & $\begin{array}{l}\text { Treatment received at } \\
\text { enrolment }\end{array}$ & Pregnancy and outcome description \\
\hline $\begin{array}{l}\text { Bhattacharya-2007 } \\
{[119]}\end{array}$ & 2 & Miltefosine & $\begin{array}{l}\text { "Despite extensive counselling for contraception, } 2 \text { cases of pregnancy were reported, with the } \\
\text { conception date close to the exposure period. One patient became pregnant } 2 \text { weeks after the end } \\
\text { of treatment, and the other became pregnant at } 3 \text { months after the end of the treatment period. } \\
\text { Two healthy babies were delivered at gestational weeks of } 39 \text { and } 40 \text {, without any birth anomaly" }\end{array}$ \\
\hline Sinha-2011 [60] & 1 & Paromomycin & $\begin{array}{l}\text { One female patient became pregnant more than } 1 \text { month after completing treatment. The } \\
\text { offspring was born alive and determined to be normal/healthy just after birth. }\end{array}$ \\
\hline Mondal-2014 [120] & 4 & $\begin{array}{l}\text { Liposomal amphotericin B } \\
\text { (single dose) }\end{array}$ & $\begin{array}{l}\text { Four female participants became pregnant within months after treatment. In one, the pregnancy } \\
\text { was completed with delivery of a term/normal birth after } 6 \text { months of follow-up. The other three } \\
\text { pregnant women were clinically healthy during the last follow-up visit. }\end{array}$ \\
\hline Jamil-2015 [121] & 1 & Paromomycin & $\begin{array}{l}\text { Pregnancy was reported in one female during the follow-up period. The offspring was born } \\
\text { healthy and a hearing test conducted on the infant at } 1.5 \text { months of age confirmed reaction to } \\
\text { sound. An otoscopy and oto-acoustic emission test to determine function of the middle and inner } \\
\text { ear was conducted at } 3 \text { months of age and confirmed normal hearing function. }\end{array}$ \\
\hline Pandey-2016 [122] & 15 & Miltefosine & $\begin{array}{l}\text { Fifteen patients became pregnant within } 6 \text { months of follow-up (all these patients became } \\
\text { pregnant } 2 \text { months after end of the treatment). All of them were followed for one year and all had } \\
\text { full-term normal pregnancy with no congenital anomalies. }\end{array}$ \\
\hline
\end{tabular}




\section{Conclusions}

In conclusion, this review brings together scattered observations on VL in pregnant women and the cases of vertically transmitted VL reported in the clinical literature. Available reports clearly underestimate the scale of the problem. Existing therapeutic guidelines regarding the usage of drugs in pregnancy is guided by limited evidence generated from case reports and small case series. Our review suggests that liposomal amphotericin B should be the preferred treatment for VL during pregnancy.

\section{Supporting information}

S1 Text. PRISMA checklist. (DOCX)

S2 Text. Search details.

(DOCX)

\section{S1 Data. Screening list.}

(XLSX)

S2 Data. Study data.

(XLSX)

S1 Table. Risk of bias assessment.

(XLSX)

\section{Acknowledgments}

We would like to thank for the Prof. Bernhard Lämmle and his team for helpful responses on queries related to their manuscript. We would like to thank Christina Woodward for locating some of the articles included in the review.

\section{Author Contributions}

Conceptualization: Prabin Dahal, Philippe J. Guerin, Piero L. Olliaro.

Data curation: Prabin Dahal, Sauman Singh-Phulgenda.

Formal analysis: Prabin Dahal, Sauman Singh-Phulgenda, Philippe J. Guerin, Piero L. Olliaro. Funding acquisition: Philippe J. Guerin.

Investigation: Prabin Dahal, Sauman Singh-Phulgenda, Philippe J. Guerin, Piero L. Olliaro.

Methodology: Prabin Dahal, Sauman Singh-Phulgenda, Brittany J. Maguire, Eli Harriss.

Project administration: Prabin Dahal, Piero L. Olliaro.

Resources: Philippe J. Guerin, Piero L. Olliaro.

Software: Prabin Dahal.

Supervision: Philippe J. Guerin, Piero L. Olliaro.

Validation: Prabin Dahal, Sauman Singh-Phulgenda, Philippe J. Guerin, Piero L. Olliaro.

Visualization: Prabin Dahal.

Writing - original draft: Prabin Dahal, Piero L. Olliaro. 
Writing - review \& editing: Prabin Dahal, Sauman Singh-Phulgenda, Brittany J. Maguire, Eli Harriss, Koert Ritmeijer, Fabiana Alves, Philippe J. Guerin, Piero L. Olliaro.

\section{References}

1. Burza S, Croft SL, Boelaert M. Leishmaniasis. Lancet. 2018; 392:951-70. https://doi.org/10.1016/ S0140-6736(18)31204-2 PMID: 30126638

2. WHO. Leishmaniasis: Key facts [Internet]. WHO. 2020 [cited 2020 Jul 5]. Available from: https://www. who.int/news-room/fact-sheets/detail/leishmaniasis

3. World Health Organization. The Global Health Observatory: Number of cases of visceral leishmaniasis reported [Internet]. 2021 [cited 2021 Jun 8]. Available from: https://www.who.int/data/gho/data/ indicators/indicator-details/GHO/number-of-cases-of-visceral-leishmaniasis-reported

4. Thakur CP, Kumar M, Pandey AK. Comparisons of regimens of treatment of antimony-resistant KalaAzar Patients: A randomized study. Am. J. Trop. Med. Hyg. 1991; 45. https://doi.org/10.4269/ajtmh. 1991.45.435 PMID: 1659239

5. Thakur CP, Singh RK, Hassan SM, Kumar R, Narain S, Kumar A. Amphotericin B deoxycholate treatment of visceral leishmaniasis with newer modes of administration and precautions: A study of 938 cases. Trans. R. Soc. Trop. Med. Hyg. 1999; 93:319-23. https://doi.org/10.1016/s0035-9203(99) 90037-8 PMID: 10492770

6. Thakur CP, Kumar A, Mitra DK, Roy A, Sinha AK, Ranjan A. Improving outcome of treatment of KalaAzar by supplementation of amphotericin B with physiologic saline and potassium chloride. Am. J. Trop. Med. Hyg. 2010; 83:1040-3. https://doi.org/10.4269/ajtmh.2010.10-0255 PMID: 21036834

7. Moore E, O'Flaherty D, Heuvelmans H, Seaman J, Veeken H, De Wit S, et al. Comparison of generic and proprietary sodium stibogluconate for the treatment of visceral leishmaniasis in Kenya. Bull. World Health Organ. 2001; 79:388-93. PMID: 11417033

8. Dietze R, Milan E, Berman J, Grogl M, et al. Treatment of Brazilian kala-azar with a short course of amphocil (amphotericin B cholesterol dispersion). Clin. Infect. Dis. 1993; 17:981-6. https://doi.org/10. 1093/clinids/17.6.981 PMID: 8110956

9. Cloots K, Burza S, Malaviya P, Hasker E, Kansal S, Mollett G, et al. Male predominance in reported Visceral Leishmaniasis cases: Nature or nurture? A comparison of population-based with health facility-reported data. PLoS Negl. Trop. Dis. 2020; 40:e0007995. https://doi.org/10.1371/journal.pntd. 0007995 PMID: 31995564

10. Dahal P, Singh-Phulgenda S, Olliaro PL, Guerin PJ. Gender disparity in patients enrolled in clinical trials of visceral leishmaniasis: a systematic review and meta-analysis. PLoS Negl. Trop. Dis. 2021; 15 : e0009204. https://doi.org/10.1371/journal.pntd.0009204 PMID: 33725005

11. Pekelharing JE, Gatluak F, Harrison T, Maldonado F, Siddiqui R, Ritmeijer K. Outcomes of visceral leishmaniasis in pregnancy: A retrospective cohort study from South Sudan. PLoS Negl. Trop. Dis. 2020; 14:e0007992. https://doi.org/10.1371/journal.pntd.0007992 PMID: 31978116

12. Adam GK, Abdulla MA, Ahmed AA, Adam I. Maternal and perinatal outcomes of visceral leishmaniasis (kala-azar) treated with sodium stibogluconate in eastern Sudan. Int. J. Gynecol. Obstet. 2009; 107:208-10. https://doi.org/10.1016/j.ijgo.2009.08.002 PMID: 19766208

13. World Health Organization. Control of the leishmaniases: Report of a meeting of the WHO Expert Committee on the Control of Leishmaniases, Geneva, 22-26 March 2010. World Heal. Organ. Tech. Rep. Ser. 949. 2010. [cited 2021 Aug 17]. Available from: http://apps.who.int/iris/bitstream/handle/ 10665/44412/WHO_TRS_949_eng.pdf?sequence=1isAllowed=y

14. Alvar J, Croft S, Olliaro P. Chemotherapy in the treatment and control of Leishmaniasis. Adv. Parasitol. 2006; 61:223-74. https://doi.org/10.1016/S0065-308X(05)61006-8 PMID: 16735166

15. Sundar S, Olliaro PL. Miltefosine in the treatment of leishmaniasis: Clinical evidence for informed clinical risk management. Ther. Clin. Risk Manag. 2007; 3:733-40. PMID: 18472998

16. Boelaert M, Sundar S. Leishmaniasis. In: Farrar J, Hotez PJ, Junghanss T, Kang G, Lalloo D, White NJ, editors. Manson's Trop. Dis. 23rd ed. Elsevier Health Sciences; 2013. p. 631-51.

17. Elamin A, Omer MIA. Visceral leishmaniasis in a 6-week-old infant: possible congenital transmission. Trop. Doct. 1992; 22:133-5. https://doi.org/10.1177/004947559202200326 PMID: 1641898

18. Eltoum IA, Zijlstra EE, Ali MS, Ghalib HW, Satti MMH, Eltoum B, et al. Congenital kala-azar and leishmaniasis in the placenta. Am. J. Trop. Med. Hyg. 1992; 46:57-62. https://doi.org/10.4269/ajtmh.1992. 46.57 PMID: 1536385

19. Low GC, Cooke WE. A congenital case of Kala-Azar. Lancet. 1926; 208:1209-11. https://doi.org/10. 1016/S0140-6736(01)05214-X 
20. Pagliano P, Ascione T, Di Flumeri G, Boccia G, De Caro F. Visceral leishmaniasis in immunocompromised: Diagnostic and therapeutic approach and evaluation of the recently released IDSA guidelines. Infez. Med. 2016; 24:265-71. PMID: 28011960

21. Pagliano P, Carannante N, Rossi M, Gramiccia M, Gradoni L, Faella FS, et al. Visceral leishmaniasis in pregnancy: A case series and a systematic review of the literature. J. Antimicrob. Chemother. 2005; 55:229-33. https://doi.org/10.1093/jac/dkh538 PMID: 15649998

22. Nuwayri-salti N, Khansa HF. Direct non-insect-vector transmission of leishmania parasites in mice. Int. J. Parasitol. 1985; 15:497-500. https://doi.org/10.1016/0020-7519(85)90043-8 PMID: 3840776

23. Utili R, Rambaldi A, Tripodi MF, Andreana A. Visceral leishmaniasis during pregnancy treated with meglumine antimoniate. Infection. 1995; 23:182-3. https://doi.org/10.1007/BF01793862 PMID: 7499009

24. Berger BA, Bartlett AH, Saravia NG, Galindo Sevilla N. Pathophysiology of Leishmania infection during pregnancy. Trends Parasitol. 2017; 33:935-46. https://doi.org/10.1016/j.pt.2017.08.012 PMID: 28988681

25. Kumar A, Mittal M, Prasad S. Treatment of leishmaniasis in pregnancy. Int. J. Gynecol. Obstet. 2001; 72:189-90. https://doi.org/10.1016/s0020-7292(00)00362-3 PMID: 11166754

26. Miah M, Ayaz F, Maniruzzaman M, Ahasan M, Bari S, Mawla S, et al. Kala azar in pregnancy. Mymensingh Med. J. 2010;Oct; 19:529-32. PMID: 20956894

27. Seaman J, Pryce D, Sondorp HE, Moody A, Bryceson ADM, Davidson RN. Epidemic Visceral Leishmaniasis in Sudan: A randomized trial of Aminosidine plus Sodium Stibogluconate versus Sodium Stibogluconate alone. J. Infect. Dis. 1993; 168:715-20. https://doi.org/10.1093/infdis/168.3.715 PMID: 8394861

28. Couderc-Pétry M, Eléfant E, Wasunna M, Mwinga A, Kshirsagar NA, Strub-Wourgaft N. Inclusion of women susceptible to and becoming pregnant in preregistration clinical trials in low-and middleincome countries: A proposal for neglected tropical diseases. PLoS Negl. Trop. Dis. 2020; 14:1-15. https://doi.org/10.1371/journal.pntd.0008140 PMID: 32525876

29. Banjara MR, Hirve S, Siddiqui NA, Kumar N, Kansal S, Huda MM, et al. Visceral leishmaniasis clinical management in endemic districts of India, Nepal, and Bangladesh. J. Trop. Med. 2012; 2012. https:// doi.org/10.1155/2012/126093 PMID: 22649459

30. Treatment of Leishmaniasis with Impavido® (Miltefosine): Pregnancy registry [Internet]. [cited 2021 Jul 29]. Available from: https://clinicaltrials.gov/ct2/show/NCT02427308

31. IMPAVIDO (miltefosine) pregnancy registry [Internet]. [cited 2021 Feb 9]. Available from: https://www. impavido.com/about-registry

32. Figueiró-Filho EA, Duarte G, El-Beitune P, Quintana SM, Maia TL. Visceral leishmaniasis (kala-azar) and pregnancy. Infect. Dis. Obstet. Gynecol. 2004; 12:31-40. https://doi.org/10.1080/ 1064744042000210384 PMID: 15460194

33. Silva JSF e., Galvao TF, Pereira MG, Silva MT. Treatment of American tegumentary leishmaniasis in special populations: A summary of evidence. Rev. Soc. Bras. Med. Trop. 2013; 46:669-77. https://doi org/10.1590/0037-8682-0104-2013 PMID: 24474006

34. Kip AE, Schellens JHM, Beijnen JH, Dorlo TPC. Clinical pharmacokinetics of systemically administered antileishmanial drugs. Clin. Pharmacokinet. 2018; 57:151-76. https://doi.org/10.1007/s40262017-0570-0 PMID: 28756612

35. WHO. Guidelines for diagnosis, treatment and prevention of visceral leishmaniasis in South Sudan [Internet]. WHO. [cited $2021 \mathrm{Jul}$ 28]. Available from: https://www. who.int/leishmaniasis/burden/ Guidelines_for_diagnosis_treatment_and_prevention_of_VL_in_South_Sudan.pdf

36. The electronic medicines compendium. AmBisome [Internet]. 2019 [cited 2021 Jul 28]. Available from: https://www.medicines.org.uk/emc/product/1022\#PREGNANCY

37. Ministry of Health-Republic of Kenya. Prevention, diagnosis and treatment of Visceral Leishmaniasis (Kala-Azar) in Kenya [Internet]. 2017 [cited 2019 Aug 30]. Available from: https://www.who.int/ leishmaniasis/burden/Kala_Azar_Kenya_2017.pdf?ua=1

38. WHO. Guideline for diagnosis, treatment \& prevention of leishmaniasis in Ethiopia [Internet]. WHO. 2013 [cited 2021 Jul 28]. Available from: https://www.who.int/leishmaniasis/burden/Guideline_for_ diagnosis_treatment_and_prevention_of_leishmaniasis_in_Ethiopia.pdf

39. Ministry of Health-Somali Fedral Government. Guidelines for diagnosis, treatment and prevention of visceral leishmaniasis in Somalia [Internet]. WHO. 2012 [cited 2021 Jul 28]. Available from: https:// www.who.int/leishmaniasis/burden/Guidelines_for_diagnosis_treatment_and_prevention_of_VL_in_ Somalia.pdf 
40. Federal Ministry of Health-Republic of Sudan. Manual for the diagnosis and treatment of leishmaniasis [Internet]. WHO. 2014 [cited 2021 Jul 28]. Available from: https://www.who.int/leishmaniasis/ burden/Manual_for_the_diagnosis_and_treatment_Leishmaniasis_Guideline_Sudan_2014.pdf?ua=1

41. Ministry of Health-Uganda. Guidelines for the diagnosis, treatment and prevention of visceral leishmaniasis in Uganda [Internet]. WHO. 2019 [cited 2021 Jul 28]. Available from: https://www.who.int/ leishmaniasis/burden/MOH_Uganda_Guidelines_diagnosis_treatment_prevention_VL.pdf

42. Anversa L, Tiburcio MGS, Richini-Pereira VB, Ramirez LE. Human leishmaniasis in Brazil: A general review. Rev. Assoc. Med. Bras. 2018; 64:281-9. https://doi.org/10.1590/1806-9282.64.03.281 PMID: 29641786

43. Brasília: Ministério da Saúde. Manual de vigilância e controle da leishmaniose visceral. [Internet]. 2014 [cited 2021 Jul 28]. Available from: http://www.saude.ba.gov.br/wp-content/uploads/2019/05/ 2014-Manual-de-vigilância-e-controle-da-leishmaniose-visceral.pdf

44. FDA. IMPAVIDO (miltefosine) prescribing information [Internet]. www.accessdata.fda.gov. [cited 2019 Oct 10]. Available from: https://www.accessdata.fda.gov/drugsatfda_docs/label/2014/204684s000lbl. pdf

45. Moher D, Liberati A, Tetzlaff J, Altman DG. The PRISMA Group (2009) Preferred Reporting Items for Systematic Reviews and Meta- Analyses: The PRISMA Statement. PLoS Med. 2009; 6:e1000097. https://doi.org/10.1371/journal.pmed.1000097 PMID: 19621072

46. Infectious Diseases Data Observatory. VL Surveyor [Internet]. www.iddo.org. 2020 [cited 2020 Sep 17]. Available from: https://www.iddo.org/vISurveyor/\#0

47. United Nations. Standard country or area codes for statistical use [Internet]. [cited 2018 Sep 13]. Available from: https://unstats.un.org/unsd/methodology/m49/overview/

48. R Core Team. R: A language and environment for statistical computing. R Found. Stat. Comput. Vienna, Austria. 2018

49. Murad MH, Sultan S, Haffar S, Bazerbachi F. Methodological quality and synthesis of case series and case reports. Evid. Based. Med. 2018; 23:60-3. https://doi.org/10.1136/bmjebm-2017-110853 PMID: 29420178

50. Silva Jde A, Araujo Ide M, Pavanetti LC, Okamoto LS, Dias M. [Visceral leishmaniasis and pregnancy in renal transplanted patient: case report]. J. Bras. Nefrol. 2015; 37:268-70. https://doi.org/10.5935/ 0101-2800.20150041 PMID: 26154649

51. Papageorgiou T, Pana Z, Tragiannidis A, Tsotoulidou V, Pratsiou E, Tzouvelekis G, et al. The first case of congenital leishmaiasis in a female infant in Greece. J. Paediatr. Child Health. 2010; 46:6112. https://doi.org/10.1111/j.1440-1754.2010.01862.x PMID: 20958824

52. Zinchuk A, Nadraga A. Congenital visceral leishmaniasis in Ukraine: case report. Ann. Trop. Paediatr. 2010; 30:161-4. https://doi.org/10.1179/146532810X12703902516400 PMID: 20522305

53. Argy N, Lariven S, Rideau A, Lemoine A, Bourgeois Moine A, Allal L, et al. Congenital Leishmaniasis in a newborn infant whose mother was coinfected with Leishmaniasis and HIV. J. Pediatr. Infect. Dis. Soc. 2020 Apr 30; 9(2):277-280. https://doi.org/10.1093/jpids/piz055 PMID: 31589299

54. El-Saaran AM, Harries JR. Visceral Leishmaniasis in Dubai. Trans R Soc Trop Med Hyg. 1979; 73:475. https://doi.org/10.1016/0035-9203(79)90189-5 PMID: 555083

55. Silveira BP, Sobrinho JA, Leite LF, Andrade Sales M das N, Araújo Gouveia M do S, Mathias RL, et al. Parto prematuro após uso de antimonial pentavalente: Relato de um caso. Rev. Soc. Bras. Med. Trop. 2003; 36:523-5. https://doi.org/10.1590/s0037-86822003000400016 PMID: 12937733

56. Banerji D. Possible congenital infection of Kala-Azar. J. Indian Med. Assoc. 1955;24. PMID: 14367842

57. Figueiro-Filho EA, El Beitune P, Queiroz GT, Somensi RS, Morais NO, Dorval ME, et al. Visceral leishmaniasis and pregnancy: analysis of cases reported in a central-western region of Brazil. Arch. Gynecol. Obstet. 2008; 278:13-6. https://doi.org/10.1007/s00404-007-0532-0 PMID: 18087708

58. Kimutai R, Musa AM, Njoroge S, Omollo R, Alves F, Hailu A, et al. Safety and effectiveness of Sodium Stibogluconate and Paromomycin combination for the treatment of Visceral Leishmaniasis in Eastern Africa: Results from a pharmacovigilance programme. Clin. Drug Investig. 2017; 37:259-72. https:// doi.org/10.1007/s40261-016-0481-0 PMID: 28066878

59. Mueller M, Balasegaram M, Koummuki Y, Ritmeijer K, Santana MR, Davidson R. A comparison of liposomal amphotericin B with sodium stibogluconate for the treatment of visceral leishmaniasis in pregnancy in Sudan. J. Antimicrob. Chemother. 2006; 58:811-5. https://doi.org/10.1093/jac/dkl342 PMID: 16916865

60. Sinha PK, Jha TK, Thakur CP, Nath D, Mukherjee S, Aditya AK, et al. Phase 4 pharmacovigilance trial of Paromomycin injection for the treatment of Visceral Leishmaniasis in India. J. Trop. Med. 2011; 2011:1-7. https://doi.org/10.1155/2011/645203 PMID: 22174722 
61. Bano $\mathrm{P}$, Shahab SM. A combination of sulphadiazine, trimethoprim and metronidazole or tinidazole in kala-azar. J. Assoc. Physicians India. 1994; 42:535-6. PMID: 7868522

62. Mittal V, Sehgal S, Yadav T, Singh VK. Congenital transmission of kala-azar. J Commun Dis. 1987; Jun:184-5. PMID: 3454359

63. Mueller Y, Mbulamberi DB, Odermatt P, Hoffmann A, Loutan L, Chappuis F. Risk factors for in-hospital mortality of visceral leishmaniasis patients in eastern Uganda. Trop. Med. Int. Heal. 2009; 14:910-7. https://doi.org/10.1111/j.1365-3156.2009.02305.x PMID: 19552645

64. Vieira ML, Jacobina RR, Soares NM. Visceral leishmaniasis in a pregnant teenager. Journal of Medical and Biological Sciences 2007; 6(3), 357-361. https://doi.org/10.9771/cmbio.v6i3.4398

65. Basher A, Nath PN. Transplacental transmission of visceral Leishmaniasis; Looking for the evidenceA case series [2P29]. Trop. Med. Int. Heal. 2017; 22 (Suppl. I):115-345.

66. Hindle E. Further observations on Chinese Kala-Azar. Proc. R. Soc. B Biol. Sci. 1928; 103:599-619.

67. Rees PH, Kager PA, Wellde BT, Hockmeyer WT. The response of Kenyan kala-azar to treatment with sodium stibogluconate. Am. J. Trop. Med. Hyg. 1984; 33:357-61. https://doi.org/10.4269/ajtmh.1984. 33.357 PMID: 6329010

68. Blanc C, Robert A. [5th case of congenital kala-azar]. Press. Med. 1984;Jul 7:1751. PMID: 6235503

69. Badaró R, Rocha H, Carvalho EM, Queiroz AC, Jones TC. Leishmania Donovani: An opportunistic microbe associated with progressive disease in three immunocompromised patients. Lancet. 1986; 327:647-9. https://doi.org/10.1016/s0140-6736(86)91725-3 PMID: 2869348

70. Nyakundi PM, Muigai R, Were JBO, Oster CN, Gachihi GS, Kirigi G. Congenital visceral leishmaniasis: Case report. Trans. R. Soc. Trop. Med. Hyg. 1988; 82:564. https://doi.org/10.1016/0035-9203(88) 90508-1 PMID: 2855677

71. Yadav T, Gupta H, Satteya U, Kumar R, Mittal V. Congenital kala-azar. Ann Trop Med Parasitol. 1989; Oct:535-7. https://doi.org/10.1080/00034983.1989.11812383 PMID: 2619366

72. Aggarwal P, Wali JP. Profile of Kala-azar in North India. Asia-Pacific J. Public Heal. 1991; 5:90-3. https://doi.org/10.1177/101053959100500116 PMID: 1665981

73. Thakur C, Sinha G, Sharma V, Barat $D$. The treatment of kala-azar during pregnancy. Natl. Med. J. India. 1993; 6:263-5. PMID: 7950931

74. Giri OP. Amphotericin B therapy in kala-azar. J. Indian Med. Assoc. 1993; 91(4):91-3. PMID: 8409490

75. Gradoni L, Gaeta GB, Pellizzer G, Maisto A, Scalone A. Mediterranean visceral leishmaniasis in pregnancy. Scand. J. Infect. Dis. 1994; 26:627-9. https://doi.org/10.3109/00365549409011824 PMID: $\underline{7855563}$

76. Jeronimo SMB, Oliveira RM, Mackay S, Costa RM, Sweet J, Eliana T, et al. An urban outbreak of visceral leishmaniasis in Natal, Brazil. Trans. R. Soc. Trop. Med. Hyg. 1994; 88:386-8. https://doi.org/10. 1016/0035-9203(94)90393-x PMID: 7570812

77. Sharma R, Bahl L, Goel A, Upadhaya A, Kaushik S, Sharma R, et al. Congenital kala-azar: a case report. J Commun Dis. 1996;Mar:59-61. PMID: 8778183

78. Thakur C, Kumar P, Kumar N, Singh G, Singh A, Narain S. A randomised comparison of classical mode of administration of amphotericin B with its newer modes of administration in kala-azar. J. Assoc. Physicians India. 1998; 46:779-83. PMID: 11229246

79. Meinecke CK, Schottelius J, Oskam L, Fleischer B. Congenital transmission of visceral leishmaniasis (Kala Azar) from an asymptomatic mother to her child. Pediatrics. 1999; 104:e65-e65. https://doi.org/ 10.1542/peds.104.5.e65 PMID: 10545591

80. Vianna VL, Takiya CM, de Brito-Gitirana L. Histopathologic analysis of hamster hepatocytes submitted to experimental infection with Leishmania donovani. Parasitol. Res. 2002; 88:829-36. https://doi.org/ 10.1007/s00436-001-0577-0 PMID: 12172815

81. Dereure J, Duong Thanh H, Lavabre-Bertrand T, Cartron G, Bastides F, Richard-Lenoble D, et al. Visceral leishmaniasis. Persistence of parasites in lymph nodes after clinical cure. J. Infect. 2003; 47:7781. https://doi.org/10.1016/s0163-4453(03)00002-1 PMID: 12850167

82. Caldas AJM, Costa JML, Gama MEA, Ramos EAG, Barral A. Visceral leishmaniasis in pregnancy: A case report. Acta Trop. 2003; 88:39-43. https://doi.org/10.1016/s0001-706x(03)00160-8 PMID: 12943975

83. Pagliano P, Rossi M, Rescigno C, Altieri S, Coppola MG, Gramiccia M, et al. Mediterranean visceral leishmaniasis in HIV-negative adults: A retrospective analysis of 64 consecutive cases (1995-2001). J. Antimicrob. Chemother. 2003; 52:264-8. https://doi.org/10.1093/jac/dkg340 PMID: 12865389

84. Kumar P V., Daneshbod Y, Sadeghipoor A. Leishmania in the glomerulus. Arch. Pathol. Lab. Med. 2004; 128:935-6. https://doi.org/10.5858/2004-128-935-LITG PMID: 15270599 
85. Figueiró Filho EA, Uehara SNO, Senefonte FR de A, Lopes AHA, Duarte G, El Beitune P. Leishmaniose visceral e gestação: relato de caso. Rev. Bras. Ginecol. e Obs. 2005; 27:92-7.

86. Boehme C, Hain U, Novosel A, Eichenlaub S, Fleischmann E, Loscher T. Congenital visceral leishmaniasis. Emerg. Infect. Dis. 2006; 12:359-3. https://doi.org/10.3201/eid1202.050449d PMID: 17080586

87. Mueller M, Ritmeijer K, Balasegaram M, Koummuki Y, Santana MR, Davidson R. Unresponsiveness to AmBisome in some Sudanese patients with kala-azar. Trans. R. Soc. Trop. Med. Hyg. 2007; 101:19-24. https://doi.org/10.1016/j.trstmh.2006.02.005 PMID: 16730363

88. Topno RK, Pandey K, Das VNR, Kumar N, Bimal S, Verma RB, et al. Visceral leishmaniasis in pregnancy -the role of amphotericin B. Ann. Trop. Med. Parasitol. 2008; 102:267-70. https://doi.org/10. 1179/136485908X278766 PMID: 18348781

89. Lorenzi A, Williams C, Griffiths I. Visceral leishmaniasis mimicking disease activity in mixed connective tissue disease. Rheumatology. 2008; 47:737-8. https://doi.org/10.1093/rheumatology/ken072 PMID: 18337264

90. Sinha PK, Roddy P, Palma PP, Kociejowski A, Lima MA, Das VNR, et al. Effectiveness and safety of liposomal amphotericin b for visceral leishmaniasis under routine program conditions in Bihar, India. Am. J. Trop. Med. Hyg. 2010; 83:357-64. https://doi.org/10.4269/ajtmh.2010.10-0156 PMID: 20682882

91. Haque MA, Ekram ARMS, Sharmin LS, Belaluddin M, Salam MA. Congenital visceral leishmaniasis. Pakistan J. Med. Sci. 2010; 26:485-7.

92. Ritmeijer K, Ter Horst R, Chane S, Aderie EM, Piening T, Collin SM, et al. Limited effectiveness of high-dose liposomal amphotericin B (AmBisome) for treatment of visceral leishmaniasis in an Ethiopian population with high HIV prevalence. Clin. Infect. Dis. 2011; 53(12):e152-58. https://doi.org/10. 1093/cid/cir674 PMID: 22016502

93. Pilaca A, Delia Z, Pepa A, Puca E, Kraja D. Vertical transmission of the Visceral Leishmaniasis: A case report. US-China Med. Sci. 2011; 8:642-5.

94. Damodaran S, Erumbala G, Abraham D, Nirmal S. Incidence of leishmaniasis in a district general hospital. Arch. Dis. Child. 2012; 97:A253. https://doi.org/10.1136/archdischild-2012-302724.0883

95. Lima TB, Villar CR, Rodrigues MAM, Baima JP, Yamashiro FDS, Franzoni LC, Caramori CA, Silva GF, Romeiro FG, Sassaki LY. Liver biopsy for visceral leishmaniasis diagnosis in pregnancy: report of 2 cases. World J. Clin. Infect. Dis. 2013; 3(2): 20-24. https://doi.org/10.5495/wjcid.v3.i2.20

96. Mescouto-Borges MRM, Maués É, Costa DL, da Silva Pranchevicius MC, Romero GAS. Congenitally transmitted visceral leishmaniasis: Report of two Brazilian human cases. Brazilian J. Infect. Dis. 2013; 17:263-6. https://doi.org/10.1016/j.bjid.2012.10.017 PMID: 23453409

97. Milosevic S, Bogavac M, Malenkovic G, Fabri M, Ruzic M, Dugandzija T. Visceral leishmaniasis as a cause of postpartum pyrexia-Case report. Cent. Eur. J. Med. 2013; 8:149-52. https://doi.org/10. 2478/s11536-012-0104-y

98. Salih NAW, van Griensven J, Chappuis F, Antierens A, Mumina A, Hammam O, et al. Liposomal amphotericin B for complicated visceral leishmaniasis (kala-azar) in eastern Sudan: How effective is treatment for this neglected disease? Trop. Med. Int. Heal. 2014; 19:146-52. https://doi.org/10.1111/ tmi.12238 PMID: 24433217

99. Burza S, Sinha PK, Mahajan R, Lima MA, Mitra G, Verma N, et al. Five-year field results and longterm effectiveness of $20 \mathrm{mg} / \mathrm{kg}$ Liposomal Amphotericin B (Ambisome) for Visceral Leishmaniasis in Bihar, India. PLoS Negl. Trop. Dis. 2014; 8:46. https://doi.org/10.1371/journal.pntd.0002603 PMID: 24392168

100. Bode SFN, Bogdan C, Beutel K, Behnisch W, Greiner J, Henning S, et al. Hemophagocytic lymphohistiocytosis in imported pediatric visceral leishmaniasis in a nonendemic area. J. Pediatr. 2014; 165:147-153.e1. https://doi.org/10.1016/j.jpeds.2014.03.047 PMID: 24797953

101. Chiverto Llamazares Y, Cabezas Lopez E, Castro Sanchez M, Iglesias Goy E. Visceral leishmaniasis as a diagnosis of puerperal fever of unknown origin. [Spanish]. Progresos Obstet. y Ginecol. 2014; 57:247-50. https://doi.org/10.1016/j.pog.2014.01.005

102. Rahman KM, Olsen A, Harley D, Butler CD, Mondal D, Luby SP, et al. Kala-azar in pregnancy in Mymensingh, Bangladesh: A social autopsy. PLoS Negl. Trop. Dis. 2014; 8:e2710. https://doi.org/10. 1371/journal.pntd.0002710 PMID: 24786280

103. Colomba C, Adamoli L, Trizzino M, Siracusa L, Bonura S, Tolomeo M, et al. A case of visceral leishmaniasis and pulmonary tuberculosis in a post-partum woman. Int. J. Infect. Dis. International Society for Infectious Diseases; 2015; 33:e5-6. https://doi.org/10.1016/j.ijid.2014.12.020 PMID: 25522871 
104. Pawar S, Ragesh R, Nischal N, Sharma S, Panda PK, Sharma SK. Unique triad of pregnancy, kala azar and hemophagocytic lymphohistiocytic syndrome from a non-endemic region. J. Assoc. Physicians India. 2015; 63:65-8. PMID: 26710404

105. Kumar R, Kumari S, Prakash J, Kumar R. Atypical presentations of visceral leishmaniasis: A case series and review of literature. Trop. J. Med. Res. 2015; 18:109-12.

106. Panagopoulos P, Mitsopoulos V, Papadopoulos A, Theodorou S, Christodoulaki C, Aloupogiannis K, et al. Visceral leishmaniasis during pregnancy: A rare case report from Greece. PLoS Negl. Trop. Dis. 2017; 11:e0005134. https://doi.org/10.1371/journal.pntd.0005134 PMID: 28207741

107. Adam GK, Omar SM, Ahmed MAA, Abdallah TM, Ali AAA. Cross-sectional study of the case-fatality rate among patients with visceral leishmaniasis infections during pregnancy in Sudan. Int. J. Gynecol. Obstet. 2018; 140:119-20. https://doi.org/10.1002/ijgo.12332 PMID: 28960291

108. Goyal V, Mahajan R, Pandey K, Singh SN, Singh RS, Strub-Wourgaft N, et al. Field safety and effectiveness of new visceral leishmaniasis treatment regimens within public health facilities in Bihar, India. PLoS Negl. Trop. Dis. 2018; 12:e0006830. https://doi.org/10.1371/journal.pntd.0006830 PMID: 30346949

109. Russo A, Alt F, Neu MA, Eder S, Wingerter A, Malki KE, et al. Hemophagocytic lymphohistiocytosis in early infancy-pitfall of differentiation between hereditary and infectious reasons. Blood. Conf. 60th Annu. Meet. Am. Soc. Hematol. ASH. 2018;132. https://doi.org/10.1182/blood-2018-99-117519

110. Cunha FT, Lopes IC, Oliveira FCS, Queiroz IT. Visceral leishmaniasis in pregnant women from Rio Grande do Norte, Brazil: A case report and literature review. Rev. Soc. Bras. Med. Trop. 2019; 52:10 2. https://doi.org/10.1590/0037-8682-0233-2018

111. Parise ÉV, Maia FSC, Gomes NSG, Silva ACP da. Óbito por leishmaniose visceral em puérpera no município de Palmas, Tocantins, Brasil. J. Heal. Biol. Sci. 2019; 7:312-9. https://doi.org/10.12662/ 2317-3076jhbs.v7i3.2149.p312-319.2019

112. Napier LE. Kala-Azar. Princ. Pract. Trop. Med. New York, USA: The Macmillan Company; 1946. p. 141.

113. Napier LE, Das Gupta CR. Indian Kala-Azar in a newly-born child. Ind. Med. Gaz. 1928;April:199-200. PMID: 29011456

114. Ahluwalia IB, Bern C, Wagatsuma $Y$, Costa $C$, Chowdhury R, Ali M, et al. Visceral Leishmaniasis: Consequences to Women in a Bangladeshi Community. J. Women's Heal. 2004; 13:360-4. https://doi. org/10.1089/154099904323087024 PMID: 15186651

115. Office of the director of Census. Census of India 2011 [Internet]. 2011 [cited 2019 Sep 19]. Available from: http://censusindia.gov.in/2011-prov-results/data_files/bihar/Provisional Population Totals 2011Bihar.pdf

116. UNESCO. Ethiopia [Internet]. 2017 [cited 2019 Sep 19]. Available from: http://uis.unesco.org/country/ ET

117. WHO TDR. Central registry for epidemiological surveillance of drug safety in pregnancy [Internet]. WHO. 2019 [cited 2019 Sep 19]. Available from: https://www.who.int/tdr/research/tb_hiv/drug-safetypregnancy/en/

118. Infectious Diseases Data Observatory. Visceral Leishmaniasis: Contributing data [Internet]. [cited 2021 Feb 12]. Available from: https://www.iddo.org/vl/data-sharing/contributing-data

119. Bhattacharya SK, Sinha PK, Sundar S, Thakur CP, Jha TK, Pandey K, et al. Phase 4 Trial of Miltefosine for the Treatment of Indian Visceral Leishmaniasis. J. Infect. Dis. 2007; 196:591-8. https://doi. org/10.1086/519690 PMID: 17624846

120. Mondal D, Alvar J, Hasnain MG, Hossain MS, Ghosh D, Huda MM, et al. Efficacy and safety of singledose liposomal amphotericin $B$ for visceral leishmaniasis in a rural public hospital in Bangladesh: $A$ feasibility study. Lancet Glob. Heal. 2014; 2:e51-7. https://doi.org/10.1016/S2214-109X(13)70118-9 PMID: 25104636

121. Jamil KM, Haque R, Rahman R, Faiz MA, Bhuiyan ATMRH, Kumar A, et al. Effectiveness study of Paromomycin IM injection (PMIM) for the treatment of Visceral Leishmaniasis (VL) in Bangladesh. PLoS Negl. Trop. Dis. 2015; 99(10): e0004118. https://doi.org/10.1371/journal.pntd.0004118 PMID: 26496648

122. Pandey K, Ravidas V, Siddiqui NA, Sinha SK, Verma RB, Singh TP, et al. Pharmacovigilance of miltefosine in treatment of visceral leishmaniasis in endemic areas of Bihar, India. Am. J. Trop. Med. Hyg. 2016; 95:1100-5. https://doi.org/10.4269/ajtmh.16-0242 PMID: 27645786

123. Saito M, Gilder ME, Nosten F, Guérin PJ, McGready R. Methodology of assessment and reporting of safety in anti-malarial treatment efficacy studies of uncomplicated falciparum malaria in pregnancy: A systematic literature review. Malar. J. 2017; 16, 491. https://doi.org/10.1186/s12936-017-2136-X PMID: 29254487 